\title{
Determination of the 3D structure of an EUV-filament observed by SoHO/CDS, SoHO/SUMER and VTT/MSDP
}

\author{
P. Schwartz ${ }^{1}$, P. Heinzel ${ }^{1}$, U. Anzer ${ }^{2}$, and B. Schmieder ${ }^{3,4}$ \\ 1 Astronomical Institute, Academy of Sciences of the Czech Republic, 25165 Ondřejov, Czech Republic \\ e-mail: pheinzel@asu.cas.cz \\ 2 Max-Planck-Institut für Astrophysik, Karl-Schwarzschild-Strasse 1, 85740 Garching, Germany \\ e-mail: ula@mpa-garching.mpg.de \\ 3 Observatoire de Paris, Section Meudon, LESIA, 92195 Meudon Principal Cedex, France \\ e-mail: brigitte.schmieder@obspm.fr \\ ${ }^{4}$ Institute of Theoretical Astrophysics, University of Oslo, Blindern, 0315 Oslo, Norway
}

Received 15 August 2003 / Accepted 14 February 2004

\begin{abstract}
Heinzel et al. (2001) found that filaments seem to be much more extended in EUV spectral lines than in H $\alpha$. In this paper we reconstruct the 3D structure of the extensions of the EUV-filament observed on 15 October 1999 by SoHO/CDS and SoHO/SUMER. Small H $\alpha$ structures were observed by VTT/MSDP. We computed the bottom and top heights of the EUV-filament extensions for several values of the hydrogen Lyman-continuum optical thickness $\tau_{912}$ using the spectroscopic model of Heinzel et al. (2003a). For this we used CDS observations of the two coronal lines Mg X $624.94 \AA$ and Si XII $520.60 \AA$. Further, we develop a new method for estimating $\tau_{912}$ using the O V line measured by CDS and the O VI measured by SUMER onboard SoHO. The latter line is used as a proxy to deduce the intensity variations of O v underneath the filament. This method also takes into account the presence of prominence-corona transition regions.

Resulting heights of the EUV-filament extensions are around $15000 \mathrm{~km}$ for the bottom and around $40000 \mathrm{~km}$ for the top boundaries. However, there are significant variations around these representative values and we show that in about half of observed pixels the Lyman-continuum absorption dominates in the coronal-line depression, while in the other half it is the volume blocking effect. The latter situation corresponds to a large (larger than $30000 \mathrm{~km}$ ) geometrical thickness of the EUV-filament extension. From the heights of the boundaries of the EUV-filament the geometrical thickness can be computed and used to estimate the total mass loading which was found to be comparable to that of typical CMEs.
\end{abstract}

Key words. Sun: filaments - radiative transfer - techniques: spectroscopic - methods: data analysis

\section{Introduction}

As found by Heinzel et al. (2001) and Schmieder et al. (2003), solar filaments seem to be more extended in EUV lines than in $\mathrm{H} \alpha$. The whole EUV-filament consists of very dark parts which are co-spatial with the $\mathrm{H} \alpha$ filament and of less dark extensions seen only in EUV but not in $\mathrm{H} \alpha$. Dark EUV structures are normally explained by the absorption of the EUV line radiation by hydrogen Lyman continuum within the cool filament body (for references see Heinzel et al. 2001). To be absorbed, the radiation of a particular EUV line must be emitted in the region between the Sun's surface and the lower boundary of the cool filament, i.e. we can consider either transition-region or coronal lines which have wavelengths below the hydrogen Lyman-continuum head $912 \AA$. The extended parts of the filament are not visible in $\mathrm{H} \alpha$ because of very low contrast which is due to the small $(\lessgtr 0.1)$ optical thickness of the $\mathrm{H} \alpha$ line. For the same column density the optical thickness $\tau_{912}$ of the hydrogen Lyman continuum is much larger (by one to two orders

Send offprint requests to: P. Schwartz,

e-mail: schwartz@asu.cas.cz of magnitude) than that of $\mathrm{H} \alpha$ which leads to observable contrast in the EUV lines due to the above-mentioned absorption mechanism (Heinzel et al. 2001). The extended parts which are visible in EUV but not in $\mathrm{H} \alpha$ are referred to as "EUV-filament extensions" (or simply EUV-extensions). Various properties of these structures are described in a review by Heinzel et al. (2003b), where different aspects of their nature are critically evaluated.

Aulanier \& Schmieder (2002) suggested that the structures producing the EUV-filament extensions are low-lying (below $4000 \mathrm{~km}$ ) cool condensations and their appearance as dark regions is entirely due to absorption of EUV-line radiation by Lyman continuum. On the other hand, Anzer \& Heinzel (2003) considered a scenario which assumes that EUV-filament extensions are structures located at higher altitudes and the reduction in intensity is caused both by absorption and the so-called volume blocking (coronal lines are not emitted from the volume occupied by cool filament plasma).

To discriminate between these two scenarios, Heinzel et al. (2003a) developed a spectroscopic model for the computation of heights of the lower and upper boundaries of the 
EUV-filament extensions. The model considers the absorption and volume blocking in two coronal lines which have different scale heights, e.g. MgX $624.94 \AA$ and Si XII $520.60 \AA$ as observed by the Coronal Diagnostic Spectrometer (CDS) (Harrison et al. 1995) onboard of the Solar and Heliospherical Observatory (SoHO). From this the altitude of the lower and upper boundary of the EUV-extension can be computed. This procedure was applied to one representative measurement within the EUV-filament extension which was part of the filament observed on May 5, 2000 (the same filament as studied by Heinzel et al. 2001; Aulanier \& Schmieder 2002; Schmieder et al. 2003). For the reasons explained below, the authors have considered $\tau_{912}$ as a free parameter and for its range between 3.8 and 8.4 they obtained the altitudes of upper and lower boundaries of the EUV-filament extension in the range 35000 to $57000 \mathrm{~km}$ and 29000 to $41000 \mathrm{~km}$, respectively. Note that the top altitude of the $\mathrm{H} \alpha$ counterpart can be also estimated by using this spectroscopic model.

The principal aim of this paper is to compute heights of the top and bottom boundaries of extended EUV structures using the new model of Heinzel et al. (2003a) which is now applied to the whole EUV-filament visible in the CDS raster. For this we have selected the filament observed on 15 October 1999 which has much more pronounced EUV-extensions compared to the previously studied filament of 5 May 2000. Since the $\mathrm{H} \alpha$ counterpart of the 15 October 1999 filament is rather small, a considerable part of the volume is occupied by the EUV-extensions which are the subject of our detailed investigation. On the basis of the spectroscopic model (which is somewhat modified in this paper), we construct the full 3D structure of the EUV-extensions. Our knowledge of altitudes of these extensions is crucial for the discrimination between the above-mentioned concepts of low-lying or high-lying structures which have a different magnetic origin.

Heinzel et al. (2003a) have also suggested to use a transition-region EUV line like $\mathrm{O} v$ to determine $\tau_{912}$, assuming that the intensity of such a line is affected only by the absorption by Lyman continuum. However, in their exploratory modelling these authors finally used $\tau_{912}$ as a free parameter (see above) because they were unable to determine the background intesity of the $\mathrm{O} v$ line emitted below the filament. We return to this problem in this paper and develope a new method to estimate $\tau_{912}$ from the intensity values of the O V $629.73 \AA$ line measured by the CDS and the OVI $1031.91 \AA$ line measured by the Solar Ultraviolet Measurements of Emitted Radiation (SUMER) (Wilhelm et al. 1995) instrument onboard of SoHO. This method is based on the assumption that the radiation of these two transition-region lines is emitted only from the solar transition region and from layers at the interface between the cool filament plasma and surrounding hot corona (the so-called "prominence-corona transition region", PCTR). We found from SoHO observations that intensity distributions of these two lines in the quiet-Sun area outside the filament correlate very well. Since the radiation of the O VI line is not absorbed by the cool filament plasma (its wavelength is above the Lyman continuum head), this line can be used as a proxy to estimate the intensity variations of the $\mathrm{OV}$ line underneath the filament.

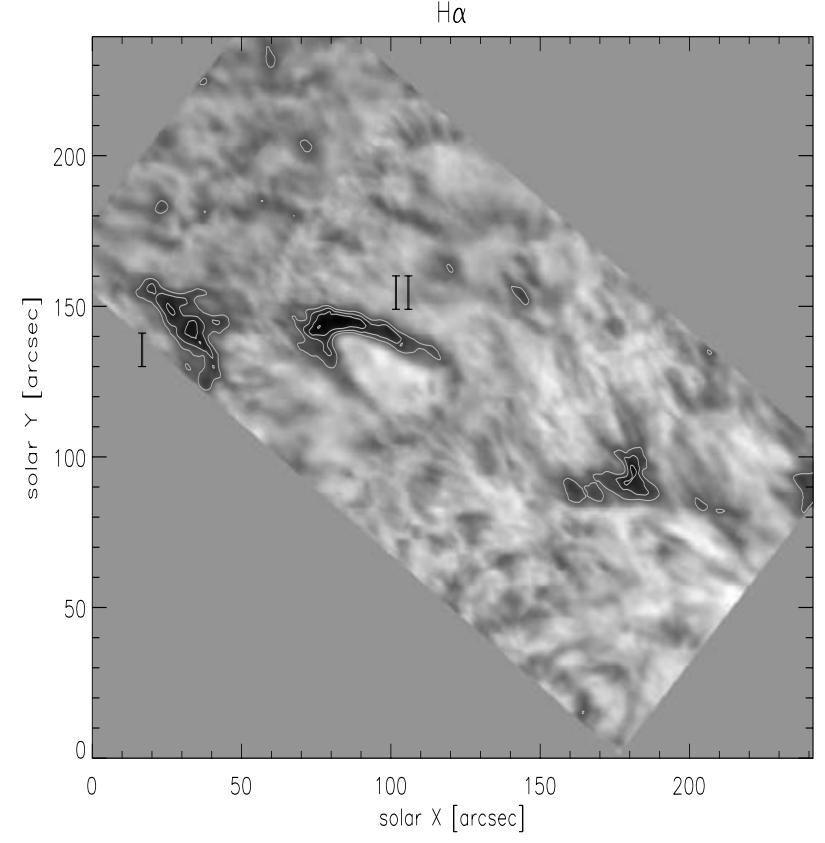

Fig. 1. H $\alpha$ observations of the filament made on 15 October 1999 by the MSDP/VTT at the time 11:27 UT.

In Sect. 2 we describe the CDS and SUMER observations of this filament. In Sect. 3 we outline the improved spectroscopic model of Heinzel et al. (2003a) and the new method for estimating $\tau_{912}$ from O V and O VI intensities. In Sect. 4 the upper and lower boundary heights of the EUV-filament extensions are computed within the whole EUV-filament area seen in the CDS raster ( $\tau_{912}$ is a free parameter in this case) and along the SUMER slit for values of $\tau_{912}$ determined by our new method from the $\mathrm{OV}$ and $\mathrm{OVI}$ line intensities. In Sect. 5 the stability of the results is discussed. Section 6 describes the general behaviour of the spectroscopic model (e.g. the existence of solutions depending on the range of scale heights of EUV lines used). Section 7 contains the discussion and the conclusions.

\section{Observations}

We used observations of the filament made on 15 October 1999 by the Multichannel Subtractive Double Pass spectrograph (MSDP) of the Vacuum Tower Telescope (VTT) on Tenerife, SoHO/CDS and SoHO/SUMER instruments. All these observations were made between 10:00 and 12:00 UT. The size and orientation of all images were adjusted according to the CDS observations.

The Carrington heliographic coordinates of the image centre are: N 37.6, E 19.3 (-253.8, 512.8 in the SoHO coordinate system). All images have dimensions $244 \operatorname{arcsec} \times 240$ arcsec.

\subsection{VTT/MSDP observations}

The filament was observed in $\mathrm{H} \alpha$ by the MSDP spectrograph on the VTT. That observation is shown in Fig. 1. Two large and very dark $\mathrm{H} \alpha$ structures marked I and II are noticeable. 

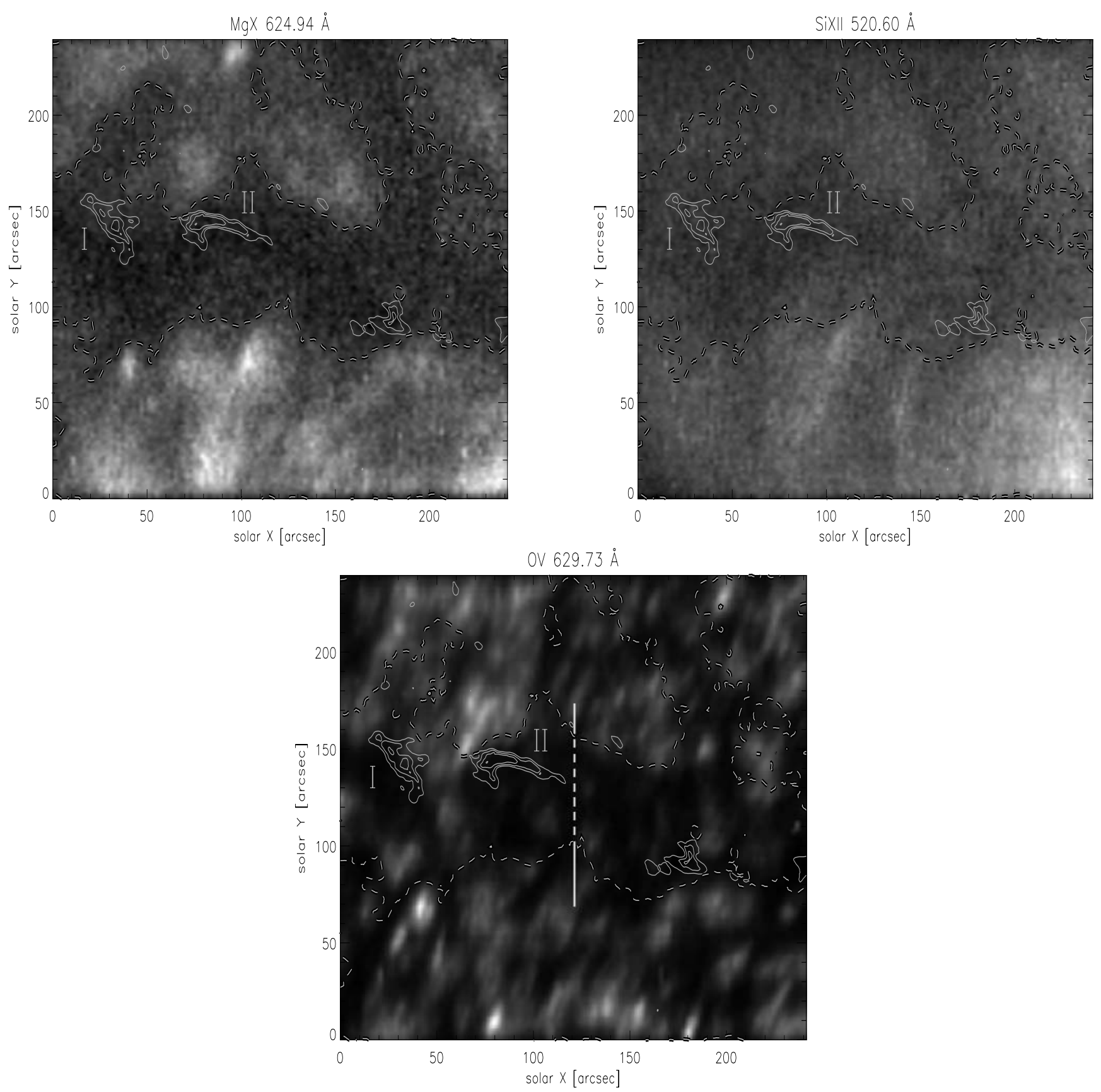

Fig. 2. Observations of the filament of 15 October 1999 in two coronal (Mg X and Si XII) and one transition-region (O V) lines made by SoHO/CDS. One can notice the depression of emission in a wide channel surrounding the $\mathrm{H} \alpha$ filament. Full-line contours represent the H $\alpha$ filament and dashed-line contours mark the EUV-filament extensions. The vertical bar shows the SUMER slit position during the O VI transitionregion line observation. More information about these images can be found in the text in Sect. 2.2.

\section{2. $\mathrm{SoHO} / \mathrm{CDS}$}

We used CDS observations of two coronal lines, Mg X $624.94 \AA$ and Si XII $520.60 \AA$ and the transition-region line O v $629.73 \AA$. These observations are shown in Fig. 2 and they are part of the Joint Observing Program 17/17718.

The observations started at 10:01:07 UT and the duration of the raster scanning was $51 \mathrm{~m} 26 \mathrm{~s}$. The $\mathrm{H} \alpha$ features are marked by full-line contours (the same as in Fig. 1). The EUV-filament extension is marked by dashed-line contours for all three panels. These contours were taken from the $\mathrm{Mg} \mathrm{X}$ observations because of lower noise in this coronal line and good visibility of the EUV-filament.

\subsection{SOHO/SUMER}

Due to the inhomogeneity in the OV line intensity distribution in the EUV-filament vicinity (bottom image of Fig. 2) it was impossible to estimate the OV line intensity distribution underneath the EUV-filament as shown in the next section. This intensity value is needed for the derivation of $\tau_{912}$. 


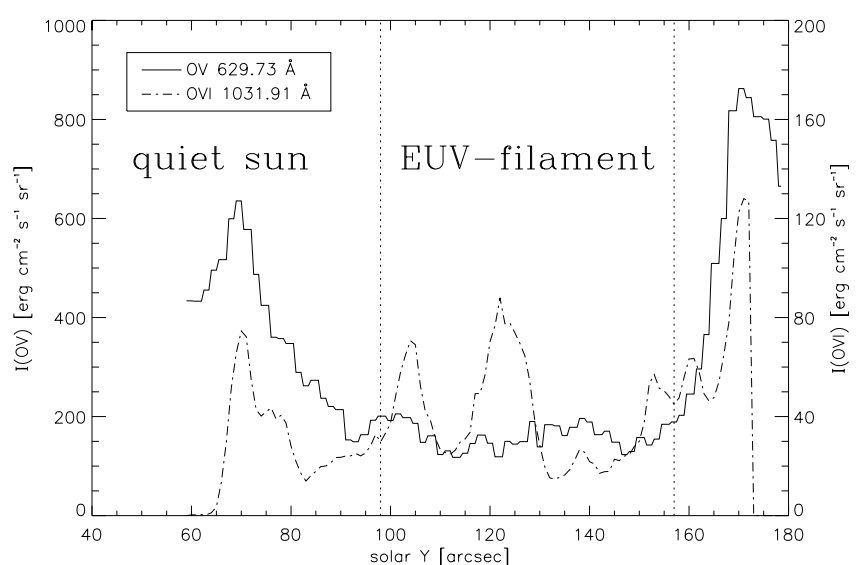

Fig. 3. SUMER observations of the O VI $1031.91 \AA$ line compared to the CDS O v $629.73 \AA$ line observations at the SUMER slit position. The graph is divided into three parts by dotted vertical lines. The left part shows data from the quiet region (EUV-filament vicinity), the middle part data across the EUV-filament and the right part data from the region where we were not sure which points belong to the quiet Sun and which to the EUV-filament.

There is also the aspect that we assume that the $\mathrm{OV}$ line is emitted only from the transition region and there is no contribution from regions in the corona above and under the EUVfilament. This seems to be justified by observations of Fludra et al. (1999) who show that the scale height of the Ov line is only a few thousand $\mathrm{km}$. This will be even smaller taking into account the uncertainty caused by the instrumental scattering (Fludra 2003). Therefore any decrease of intensity should be caused only by Lyman-continuum absorption in cool filament plasma. But it is also possible that there are some PCTRs $(\mathrm{PCTR}=$ prominence-corona transition region $)$ at the bottom and/or at the top of the EUV-filament. That makes the situation more complex. The problem can be handled by using one more transition-region oxygen ion line which is not absorbed by the EUV-filament hydrogen plasma. So we need some observation in a line with a wavelength greater than the wavelength of the Lyman-continuum head. Suitable are lines of O IV or O VI ions for which the line formation temperature is close to that of the $\mathrm{OV}$ line.

Such a spectral line is OVI $1031.91 \AA$ measured by SUMER during observation No. 5 (Fig. 3) of the observing program JOP 17/107 made at 10:55:49 UT. There were two other OVI measurements in that observing program but we used this one because of the best spatial correlation with the O V CDS measurement in the quiet-Sun regions.

The O VI line was observed with a slit 120 arcsec long and with 30 s exposure time. The position of the SUMER slit in the CDS raster is shown at the bottom image of Fig. 2 as vertical bar (CDS raster coordinates of the slit are $X=122$ arcsec, $Y=59 \div 179$ arcsec). The dashed part of this line crosses the EUV-filament and the rest of it crosses the region adjacent to the EUV-filament (quiet Sun).

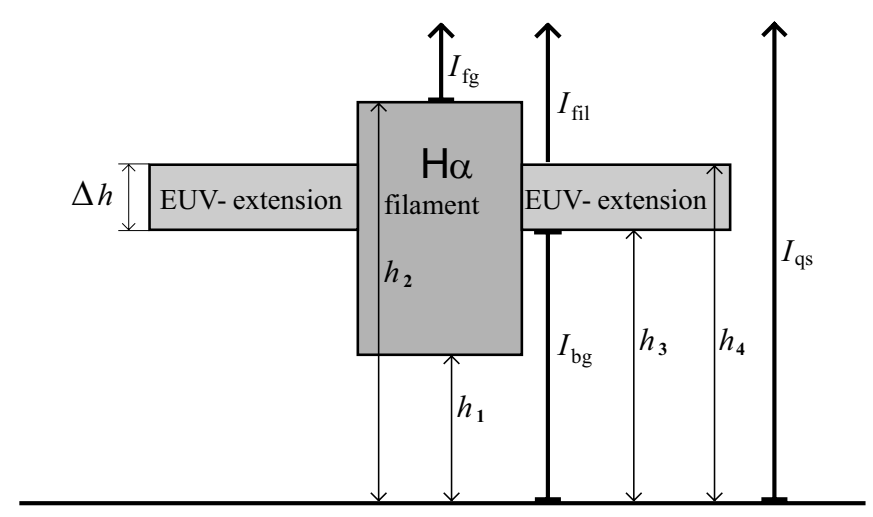

Fig. 4. Schematic drawing of the EUV-filament model designed by Heinzel et al. (2003a). For definitions of individual quantities see the text. All intensities except $I_{\text {fil }}$ are obtained by integration over the heights along the respective sections expressed by thick lines. $I_{\text {fil }}$ (see Eq. (8)) is equal to the radiation $I_{\mathrm{bg}}$ emitted from the underneath of the EUV-filament absorbed by its plasma plus the contribution from the corona above the filament.

\section{Methods of computation}

\subsection{Spectroscopic model for the computation of EUV-filament heights}

If we take $\tau_{912}$ as a free parameter (possible ways of its determination will be described in the next Subsection), the spectroscopic model of Heinzel et al. (2003a) requires EUV observations of at least two coronal lines with wavelengths shorter than $912 \AA$ (head of the hydrogen Lyman continuum).

The model is sketched in Fig. 4 where individual quantities have the following meaning:

$h_{1}$ - height of the lower boundary of the $\mathrm{H} \alpha$ part of the filament (all heights are measured from the solar surface);

$h_{2}$ - height of the upper boundary of the $\mathrm{H} \alpha$ part of the filament;

$h_{3}$ - height of the lower boundary of the EUV-filament extensions;

$h_{4}$ - height of the upper boundary of the EUV-filament extensions;

$\Delta h$ - geometrical thickness of the EUV-filament extensions $\left(\Delta h=h_{4}-h_{3}\right)$;

$I_{\mathrm{qs}}-$ quiet-Sun disk intensity, measured in the EUV-filament vicinity;

$I_{\mathrm{bg}}$ - intensity of radiation incident at the bottom surface of the EUV-filament (i.e. background intensity);

$I_{\text {fil }}$ - intensity of the EUV-filament;

$I_{\mathrm{fg}}$ - intensity measured at the darkest points within the $\mathrm{H} \alpha$ part of the filament.

This model assumes a uniform coronal radiation within the whole CDS raster area. We call this "quiet-Sun" radiation although the actual intensity can be enhanced. One also assumes a spherically symmetrical distribution of the volume emissivity of coronal lines in the "quiet-Sun" region. All intensities designed above are integrated line intensities. 
We define the function $f(h)$ :

$f(h)=\frac{1}{I_{\mathrm{qs}}} \int_{0}^{h} \varepsilon\left(h_{\mathrm{r}}\right) \mathrm{d} h^{\prime}$,

where

$I_{\mathrm{qs}}=\int_{0}^{\infty} \varepsilon\left(h_{\mathrm{r}}\right) \mathrm{d} h^{\prime}$.

The quantity $\varepsilon\left(h_{\mathrm{r}}\right)$ is the spectral line volume emissivity as function of height $h_{\mathrm{r}}$ above the surface in radial direction, $h$ and $h^{\prime}$ are heights above the surface in the line-of-sight direction.

From various coronal line observations on the limb it is known that the intensity decreases approximately exponentially with height, i.e.

$I\left(h_{\mathrm{r}}\right)=I_{\mathrm{o}} \exp \left(-h_{\mathrm{r}} / H\right)$,

where $H$ is the scale-height and $I_{0}$ is the intensity at the limb. (see e.g. Fludra et al. 1999). Assuming a spherically-symmetrical distribution of $\varepsilon\left(h_{\mathrm{r}}\right)$ and using the Abel transformation (see e.g. Griem 1997) we obtain for $H \ll$ $R_{\odot}$ the formula (Schwartz 2002):

$\varepsilon\left(h_{\mathrm{r}}\right) \approx \frac{1}{\sqrt{2 \pi}} I_{\mathrm{o}} \sqrt{\frac{1}{\left(R_{\odot}+h_{\mathrm{r}}\right)}} \exp \left(-h_{\mathrm{r}} / H\right)$.

In this case we can also write:

$f\left(h_{\mathrm{r}}\right) \approx 1-\exp \left(-h_{\mathrm{r}} / H\right)$.

Now we shall discuss a geometrical effect for filaments which are not at the disk centre. In Eqs. (1)-(5) the quantity $h_{\mathrm{r}}$ represents the height above the solar surface in the radial direction. However, all intensities used were naturally measured along the line-of-sight direction. Therefore, it is necessary to transform $h_{\mathrm{r}}$ in the above equations to the line-of-sight direction heights $h$. If the angle $\alpha$ between the line-of-sight and the radial direction at the filament position is small (less than few tens of degrees, so that the filament is not far from the centre of the solar disk) we can use a plane-parallel approximation for that transformation. It is evident from Fig. 5 that $h_{\mathrm{r}}$ transforms as:

$h_{\mathrm{r}}=h \cos \alpha$.

This can be easily included into the model of Heinzel et al. (2003a) by using the scale-height $H^{\prime}$ instead of the measured $H$ :

$H^{\prime}=\frac{H}{\cos \alpha}$

and using the height $h$ instead of $h_{\mathrm{r}}$.

For both observed coronal lines one can write (see Fig. 4):

$I_{\text {fil }}=\int_{h_{4}}^{\infty} \varepsilon\left(h^{\prime}\right) \mathrm{d} h^{\prime}+\exp \left(-\tau_{\lambda}\right) \int_{0}^{h_{3}} \varepsilon\left(h^{\prime}\right) \mathrm{d} h^{\prime}$.

Using the definition of $f(h)$ and $H^{\prime}$ instead of $H$, we finally obtain:

$I_{\text {fil }}=I_{\mathrm{qs}}\left[1-f\left(h_{4}\right)\right]+I_{\mathrm{qs}} f\left(h_{3}\right) \exp \left(-\tau_{\lambda}\right)$,

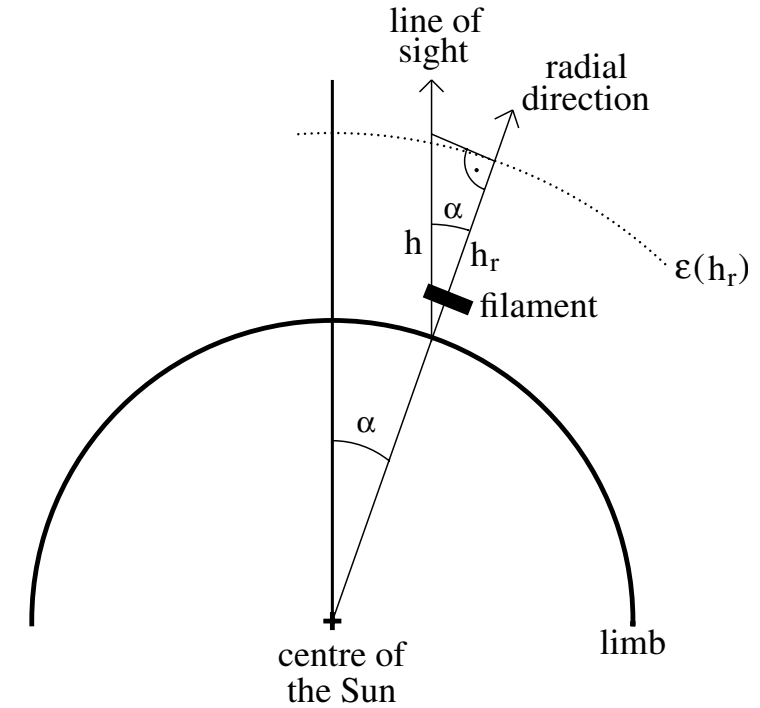

Fig. 5. Plane-parallel approximation used for the transformation between the heights in the radial and the line-of-sight directions for an angle $\alpha$ less than a few tens of degrees. The dotted line represents surfaces in the corona with a constant value of $\varepsilon\left(h_{\mathrm{r}}\right)$.

where $\tau_{\lambda}$ is the optical thickness of the Lyman continuum at the position of the coronal line. From the relation

$\frac{I_{\mathrm{fg}}}{I_{\mathrm{qs}}}=\int_{h_{2}}^{\infty} \varepsilon\left(h^{\prime}\right) \mathrm{d} h^{\prime}$

and Eq. (5) it follows that $h_{2}$ can be determined from the known $H^{\prime}(\mathrm{Mg}$ X) (Heinzel et al. 2003a):

$h_{2}=-\frac{H^{\prime}(\mathrm{Mg} \mathrm{x})}{\ln \left[1-f\left(h_{2}\right)\right]}$

Comparing the scale heights for the MgIX $368 \AA$ line derived from the measurement of a quiet-Sun region above the limb published by Fludra et al. (1999) and the one estimated from measurements of an active region published by Sterling et al. (1999) it can be assumed that the scale height for the Mg IX coronal line intensity is not much dependent on solar activity. Therefore, Heinzel et al. (2003a) assumed that also the scale height for the $\mathrm{MgX}$ line intensity is solar-activity independent and they used a value of $32000 \mathrm{~km}$ on the basis of the observations by Fludra et al. (1999). This scale-height value is used also by us but it had to be transformed to the lineof-sight direction using Eq. (7). For a known value of $h_{2}$ it is then possible to compute the scale height for Si XII line using Eqs. (5) and (10):

$H^{\prime}(\operatorname{Si}$ XII $)=-\frac{h_{2}}{\ln \left[1-f\left(h_{2}\right)\right]}$.

This value of the scale-height gives the intensity dependence on height in the line-of-sight direction.

Considering $\tau_{912}$ as a free parameter for the model of Heinzel et al. (2003a), it is necessary to compute the optical thicknesses of the Lyman continuum at the position of both coronal lines used in this model. We take the $\lambda^{3}$-dependence 
for the hydrogen Lyman-continuum opacity. Then the ratio of $\tau_{\lambda} / \tau_{912}$ is expressed as:

$$
\frac{\tau_{\lambda}}{\tau_{912}}=\frac{\lambda^{3}}{(912)^{3}} \frac{g_{\mathrm{II}}(\lambda)}{g_{\mathrm{II}}(912)}
$$

where $g_{\mathrm{II}}(\lambda)$ is the Gaunt factor which is for our coronal and transition-region lines $g_{\mathrm{II}}(\lambda) \approx 1$ and for the Lyman-continuum head $g_{\mathrm{II}}(912) \approx 0.8$.

On this basis it is possible to calculate $h_{3}$ and $h_{4}$ iteratively. We start the iteration from $h_{4}=0$, then the values of $f\left(h_{4}\right)$ are computed for both $\mathrm{MgX}$ and Si XII lines using Eq. (5). Then $f\left(h_{3}\right)$ for both lines is computed using Eq. (9) and the $h_{3}$ values from these $f\left(h_{3}\right)$ are obtained as:

$$
h_{3}=-\frac{H^{\prime}}{\ln \left[1-f\left(h_{3}\right)\right]} .
$$

If the $h_{3}$ values computed for both $\mathrm{Mg}$ X and Si XII lines differ from each other, then $h_{4}$ is increased by a prescribed step and new values of $h_{3}$ are computed. The iteration stops when the $h_{3}$ values computed for both lines differ by an amount smaller than the desired accuracy. As a result, we obtain two heights $h_{3}$ and $h_{4}$. If the resulting value of $h_{3}$ is higher than $h_{4}$, the solution is considered as unphysical. We stop the calculation also when $h_{4}$ or $h_{3}$ become larger than some prescribed value.

\subsection{Method of determination of $\tau_{912}$ from simultaneous OV and OVI TR line observations}

We a priori assume that the EUV-filament is located at heights larger than the scale heights of the O V $629.73 \AA$ and O VI $1031.91 \AA$ TR lines. So these lines are radiated only from the TR. Then the observed decrease of the $\mathrm{OV}$ line intensity due to absorption by the EUV-filament plasma should allow the $\tau_{912}$ determination. But the distribution of intensity of the $\mathrm{OV}$ line is very inhomogeneous (see bottom image of Fig. 2) in the vicinity of the EUV-filament. Therefore it was impossible to estimate a reliable intensity distribution underneath the EUV-filament. Therefore, we used SoHO/SUMER observations of the OVI TR line which is not absorbed by the EUV-filament hydrogen plasma (because of $\lambda(\mathrm{OVI})>$ $912 \AA$ ). The method also takes into account the possibility of a PCTR occurrence.

The method is based on the model which is sketched in Fig. 6. The meaning of $I_{\mathrm{fg}}, I_{\mathrm{qs}}$ and $I_{\mathrm{bg}}$ is similar to that in the previous section. Assuming that the intensities of both $\mathrm{OV}$ and O VI lines outside the EUV-filament are emitted only from the TR at the solar surface (we neglect any coronal contribution), the $I_{\mathrm{bg}}$ and $I_{\mathrm{qs}}$ intensity will be the same. Here we use two different names only for clarification: $I_{\mathrm{bg}}$ is the intensity of the TR line radiation emitted underneath the filament and $I_{\mathrm{qs}}$ is the TR line intensity outside the filament.

We assume that the ratios of the quiet-Sun intensities as well as the background intensities of both $\mathrm{OV}$ and $\mathrm{O}$ VI lines are constant and we denote them by $b$ :

$b=\frac{I_{\mathrm{qs}}(\mathrm{O} \mathrm{V})}{I_{\mathrm{qs}}(\mathrm{O} \mathrm{VI})}=\frac{I_{\mathrm{bg}}(\mathrm{O} \mathrm{V})}{I_{\mathrm{bg}}(\mathrm{O} \mathrm{VI})}$

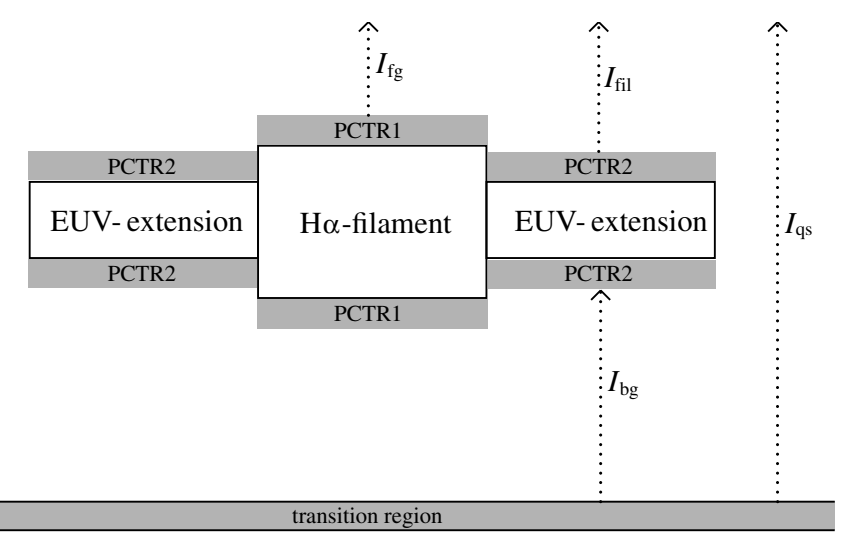

Fig. 6. Schematic drawing of the model used for the $\tau_{912}$ determination. Regions marked by PCTR1 are prominence-corona transition regions on the top and bottom of the $\mathrm{H} \alpha$ part of the filament. PCTR2 are such regions on the top and bottom of the EUV-filament extensions. Intensities emitted from different regions are marked by dotted-line arrows.

This assumption is supported by two facts. First, the ratio as determined from observations (Fig. 3), is indeed fairly constant we will return to this later. Second, the constancy of $b$ also follows from a similar temperature dependence of the intensities of both lines. The spectral line intensity can be computed using the formula (Young et al. 2003):

$I=\int A($ element $) C\left(n_{\mathrm{e}}, T\right) \Phi(T) \mathrm{d} T$,

where $A$ (element) is the element abundance, $C\left(n_{\mathrm{e}}, T\right)$ is the contribution function (depending on electron density $n_{\mathrm{e}}$ and electron temperature $T$ ) and $\Phi(T)$ is the differential emission measure. For the given temperature distribution across the TR the ratio of the $\mathrm{OV}$ and $\mathrm{OVI}$ line intensities depends only on the ratio of the contribution functions (here the scattering of line radiation is not considered - see discussion below). If the temperature dependence of the contribution functions of both $\mathrm{OV}$ and $\mathrm{O} \mathrm{VI}$ lines is similar, the intensity distribution of both lines will be also similar.

Temperature variations of the contribution functions of $\mathrm{O} \mathrm{V}$ and O VI lines were computed using the CHIANTI atomic database version 4.02 (Young et al. 2003) and are shown in Fig. 7. The contribution function is defined as:

$C\left(n_{\mathrm{e}}, T\right)=\frac{h c}{4 \pi \lambda} \frac{A_{\mathrm{ul}}}{n_{\mathrm{e}}} \frac{N_{\mathrm{u}}}{N(\text { ion })} \frac{N(\text { ion })}{N(\text { element })}$.

$A_{\mathrm{ul}}$ is the spontaneous transition probability from level $\mathrm{u}$ to level 1 and $n_{\mathrm{e}}$ is the density of free electrons. $N_{\mathrm{u}} / N$ (ion) is the temperature and electron-density dependent ratio of concentration of ions in the upper level of transition to total number of the ions. $N($ ion $) / N$ (element) is the temperature dependent ratio of the same element ion number $(\mathrm{O} V$ or $\mathrm{O}$ VI in our case) to the number of all ions of that element. Values of this ratio are computed from ionization equilibrium and we used the results of Arnaud \& Raimond (1992).

Assuming that the PCTR regions on the top and bottom of the $\mathrm{H} \alpha$ filament and EUV-extensions (i.e. PCTR1 and 


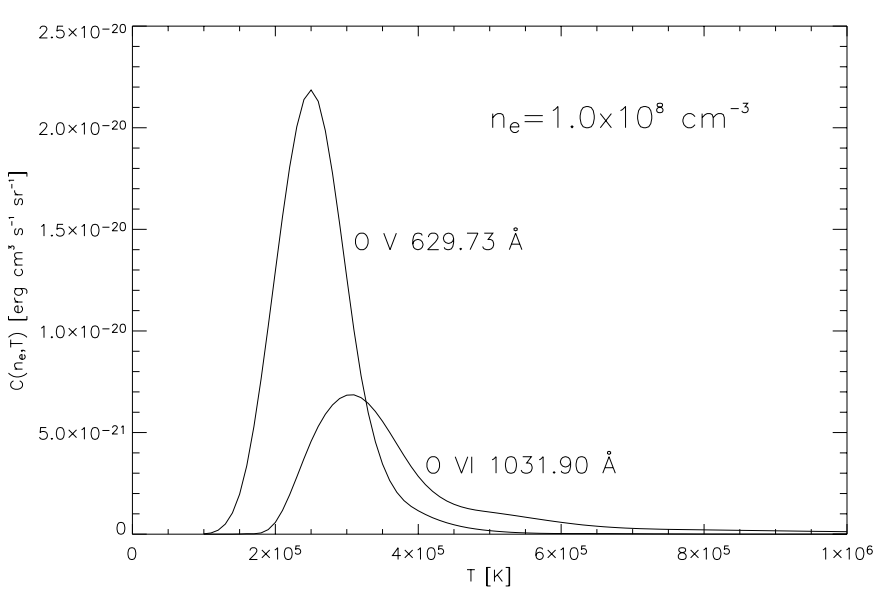

Fig. 7. Comparison of temperature dependence of the contribution functions for an electron density of $10^{8} \mathrm{~cm}^{-3}$ for both oxygen ion lines. The contribution functions were computed using the CHIANTI database version 4.02 (Young et al. 2003).

PCTR2 regions) are similar and comparable to the transition region, the following ratio holds:

$b=\frac{I_{\mathrm{PCTR} 1}(\mathrm{O} \mathrm{V})}{I_{\mathrm{PCTR} 1}(\mathrm{O} \mathrm{VI})}=\frac{I_{\mathrm{PCTR} 2}(\mathrm{O} \mathrm{V})}{I_{\mathrm{PCTR} 2}(\mathrm{O} \mathrm{VI})}$.

Our assumption about similarity of both PCTRs is based on the condition that the emissivity in these PCTRs is dominated by thermal processes at temperatures indicated in Fig. 7 and the densities are also similar in both PCTRs. However, if one would consider the scattering of background TR radiation in the $\mathrm{OV}$ and $\mathrm{OVI}$ lines, the situation will be more complicated. Such a scattering is considered e.g. for the OVI line at coronal temperatures and densities to deduce the speed of the solar wind (see Kohl et al. 1997). In the case of $\mathrm{OV}$ scattering, the bottom parts of PCTR1 and PCTR2 will be more radiatively excited than the upper parts, simply due to the Lyman-continuum absorption of background radiation by the $\mathrm{H} \alpha$ filament and the EUV-extension.

The $\mathrm{H} \alpha$ part of the filament is optically thick for the $\mathrm{OV}$ line, so

$I_{\mathrm{PCTR} 1}(\mathrm{OV})=I_{\mathrm{fg}}(\mathrm{OV})$,

where $I_{\text {PCTR1 }}$ is the intensity of the $\mathrm{OV}$ line emitted from the PCTR1 region. Because we took the PCTR1 and PCTR2 regions to be similar we can write:

$I_{\mathrm{PCTR} 2}=a I_{\mathrm{PCTR} 1}$,

where $a$ is a constant parameter which we assume to lie between 0 and 1 .

Using the model shown in Fig. 6 the OVI line intensity above the EUV-filament is given by:

$I_{\mathrm{fil}}(\mathrm{O}$ VI $)=2 I_{\mathrm{PCTR} 2}(\mathrm{O}$ VI $)+I_{\mathrm{bg}}(\mathrm{O}$ VI $)$

and from this we get:

$I_{\mathrm{fil}}(\mathrm{O} \mathrm{VI})=2 \frac{a}{b} I_{\mathrm{fg}}(\mathrm{O} \mathrm{V})+\frac{1}{b} I_{\mathrm{bg}}(\mathrm{OV})$.
Table 1. Values of the quiet-Sun and foreground intensities for three lines observed by SoHO/CDS.

\begin{tabular}{cccc}
\hline \hline line & $\begin{array}{c}\text { wavelength } \\
{[\AA]}\end{array}$ & $\begin{array}{c}I_{\mathrm{fg}} \\
{\left[\mathrm{erg} \mathrm{cm}^{-2} \mathrm{~s}^{-1} \mathrm{sr}^{-1}\right]}\end{array}$ & $\begin{array}{c}I_{\mathrm{qs}} \\
{\left[\mathrm{erg} \mathrm{cm}^{-2} \mathrm{~s}^{-1} \mathrm{sr}^{-1}\right]}\end{array}$ \\
\hline $\mathrm{Mg} \mathrm{X}$ & 624.94 & 25 & 75 \\
Si XII & 520.60 & 40 & 87 \\
O v & 629.73 & 206 & $-^{*}$ \\
\hline
\end{tabular}

* Not estimated because of the great inhomogeneity in the distribution of the $\mathrm{OV}$ line intensity within the CDS raster (see bottom image in Fig. 2).

Owing to fact that the $\mathrm{OV}$ line radiation is absorbed by the EUV-filament hydrogen plasma, we can write (see Fig. 6):

$$
\begin{aligned}
I_{\mathrm{fil}}(\mathrm{OV})= & {\left[I_{\mathrm{bg}}(\mathrm{OV})+I_{\mathrm{PCTR} 2}(\mathrm{OV})\right] \exp \left(-\tau_{\mathrm{O} \mathrm{v}}\right) } \\
& +I_{\mathrm{PCTR} 2}(\mathrm{OV}) .
\end{aligned}
$$

Finally using Eqs. (22) and (23) and the above relations we get for $\tau_{\mathrm{OV}}$ :

$\tau_{\mathrm{OV}}=-\ln \left[\frac{I_{\mathrm{fil}}(\mathrm{OV})-a I_{\mathrm{fg}}(\mathrm{OV})}{b I_{\mathrm{fil}}(\mathrm{OVI})-a I_{\mathrm{fg}}(\mathrm{OV})}\right]$.

The $\tau_{\mathrm{O} v}$ value then has to be transformed to $\tau_{912}$ using Eq. (13).

\section{Results}

\subsection{Height maps of upper and lower boundaries of the EUV-filament for $\tau_{912}$ taken as a free parameter}

Maps of upper $\left(h_{4}\right)$ and and lower $\left(h_{3}\right)$ boundaries of the EUV-filament observed on 15 October 1999 by SoHO/CDS are shown in Fig. 8 for three values of $\tau_{912}$ equal to 3,5 and 7 . Those maps were computed using the spectroscopic model of Heinzel et al. (2003a) modified as described in Sect. 3.1. For $I_{\mathrm{fg}}$ (in both $\mathrm{Mg} \mathrm{X}$ and Si XII lines) we adopted average values from the two largest and darkest $\mathrm{H} \alpha$ filament regions labeled I and II in the images in Fig. 2. We assumed a unique value of the quietSun intensity $I_{\mathrm{qs}}$ for the whole region where the EUV-filament was present. So $I_{\mathrm{qs}}$ was estimated as an average value from the EUV-filament vicinity but only from the quiet-Sun region at $Y$ ranging from 0 to 100 arcsec (in the relative CDS-raster coordinate system). This region is marked by a dashed contour in the lower part of all three images in Fig. 2. Other regions outside the EUV-filament were not used for quiet-Sun intensity estimates because we were not sure whether they belong to the EUV-filament or to the quiet-Sun. Values of $I_{\mathrm{fg}}$ and $I_{\mathrm{qs}}$ for both $\mathrm{Mg} \mathrm{X}$ and Si XII lines are listed in Table 1. To include the geometrical correction in the computations, an angle $\alpha$ between the line of sight and radial direction had to be computed using the formula for angle distance between the centre of the solar disk and the filament:

$\cos \alpha=\sin b \sin b_{\mathrm{o}}+\cos b \cos b_{\mathrm{o}} \cos \left(l-l_{\mathrm{o}}\right)$. 
$h_{3}[\mathrm{~km}] ; \quad \tau(912 \AA)=3.0$

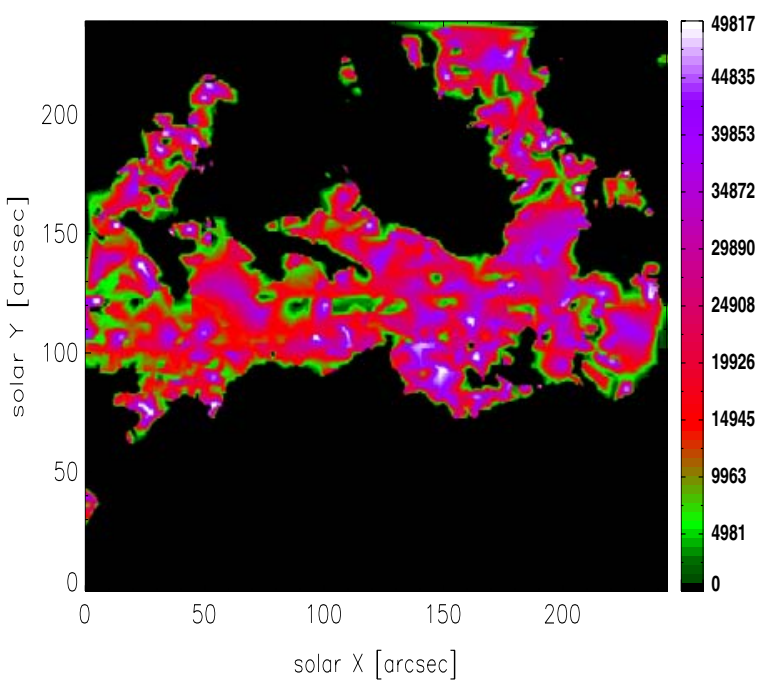

$h_{3}[\mathrm{~km}] ; \quad \tau(912 \AA)=5.0$

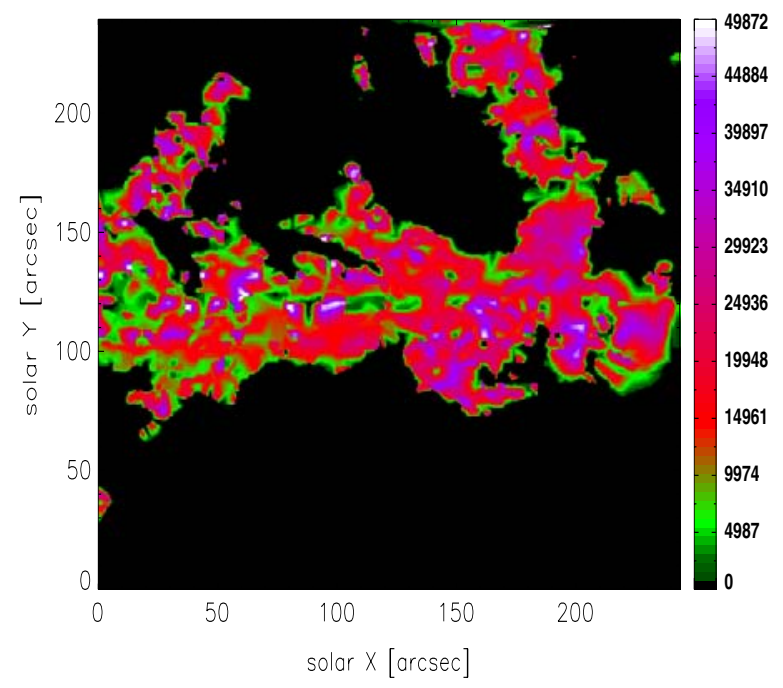

$h_{3}[\mathrm{~km}] ; \quad \tau(912 \AA)=7.0$

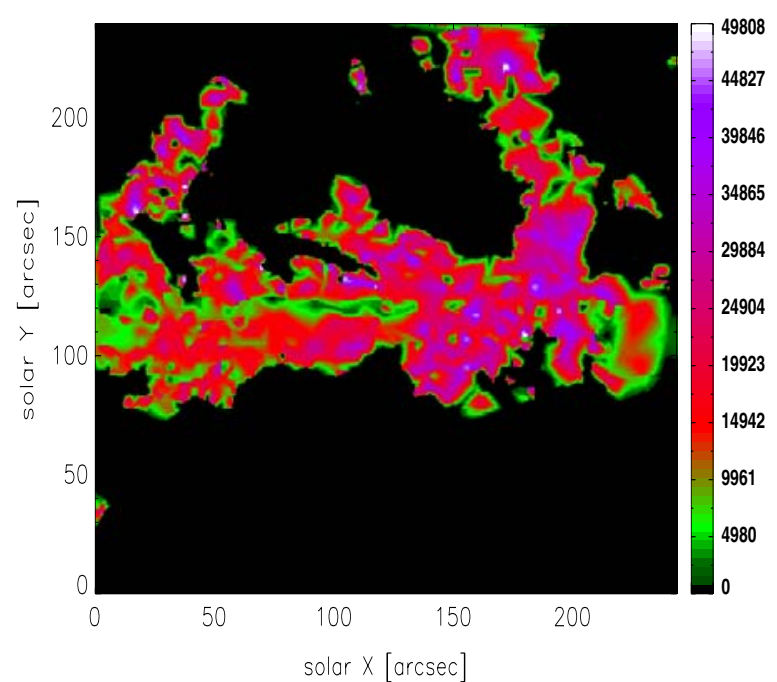

$h_{4}[\mathrm{~km}] ; \quad \tau(912 \AA)=3.0$

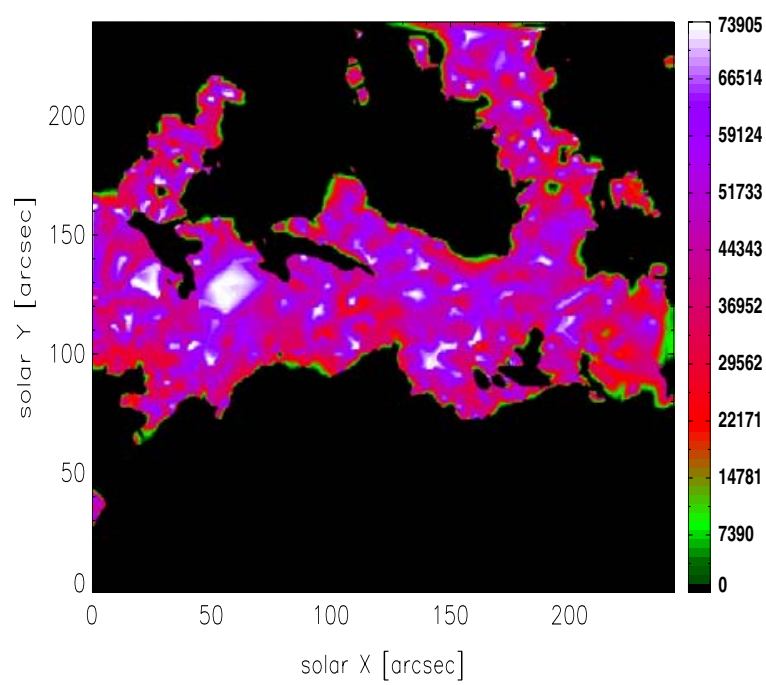

$h_{4}[\mathrm{~km}] ; \quad \tau(912 \AA)=5.0$

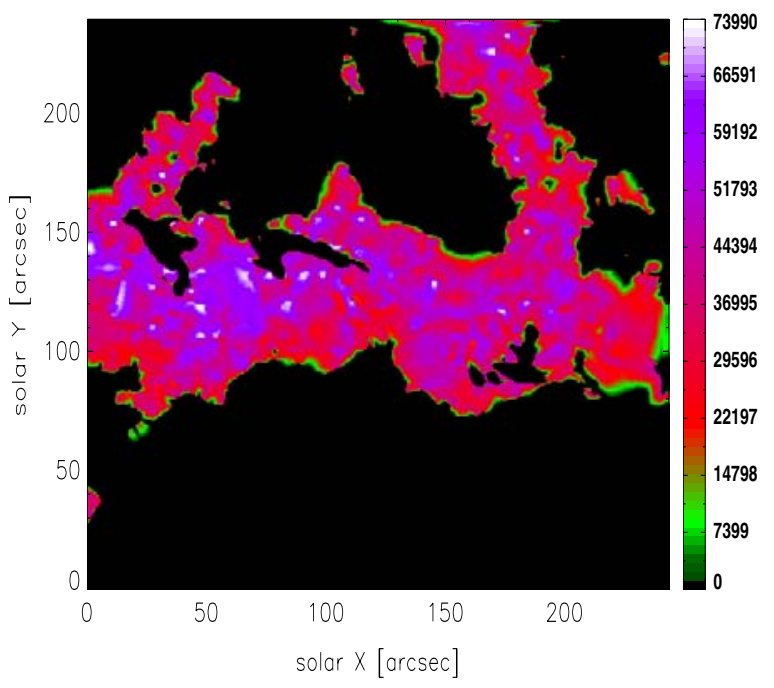

$h_{4}[\mathrm{~km}] ; \quad \tau(912 \AA)=7.0$

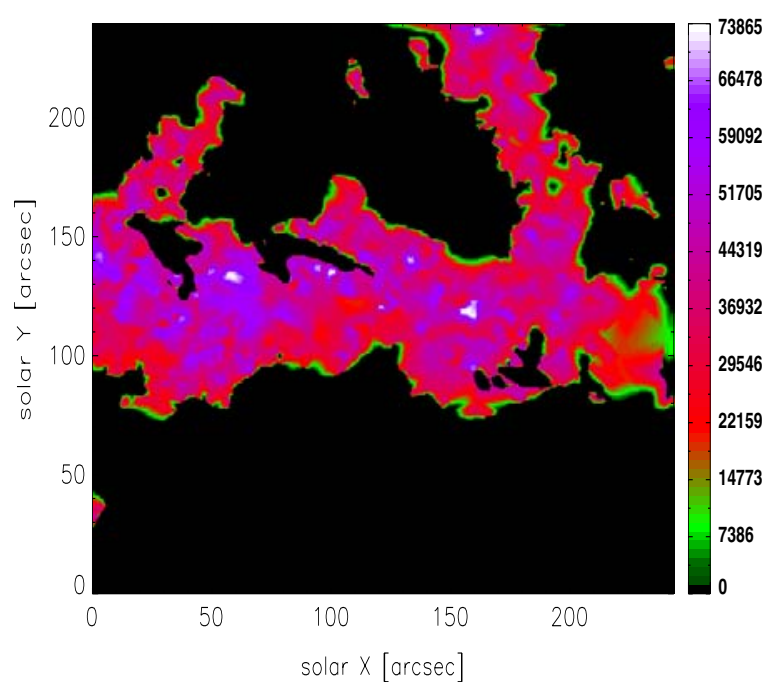

Fig. 8. Maps of the height of upper $\left(h_{4}\right)$ and lower $\left(h_{3}\right)$ boundaries of the EUV-filament extensions for $\tau_{912}$ values 3.0, 5.0 and 7.0. These maps were computed using the spectroscopic model of Heinzel et al. (2003a) modified as described in Sect. 3.1. 
Carrington heliographic coordinates of the centre of the CDS raster were: $l=\mathrm{N} 37^{\circ} .6$ and $b=\mathrm{E} 19^{\circ} .3$. Coordinates of the centre of the solar disk for 15 October 1999 were: $l_{\mathrm{o}}=0^{\circ}$ and $b_{\mathrm{o}}=5.9$. Then we compute $\alpha=38.9$. For such an angle, using the plane-parallel approximation causes an error in volume emissivity $\varepsilon(h)$ about two orders of magnitude less than its values. So, the influence of such a small error to the spectroscopical model computation is negligible.

For the $\mathrm{MgX}$ scale height in the radial direction a value of $32000 \mathrm{~km}$ was estimated from measurements published by Fludra et al. (1999). We prescribed upper limits of $50000 \mathrm{~km}$ for $h_{3}$ and $74000 \mathrm{~km}$ for $h_{4}$. These limits were selected according to low statistical significance and great scatter of computed heights above these limits. Therefore, we can suppose that the computed heights above these limits are only due to noise in the observed data.

In addition to $h_{3}$ and $h_{4}$ we computed two other quantities using the model of Heinzel et al. (2003a): $44500 \mathrm{~km}$ for the height $\left(h_{2}\right)$ of the upper boundary of the $\mathrm{H} \alpha$ part of the filament and $46100 \mathrm{~km}$ for the scale height of the Si XII line in radial direction. The value $74000 \mathrm{~km}$ for this scale height, estimated from observations published by Fludra et al. (1999), is rather different from our value but it could be due to a strong dependence of the Si XII line scale height on local solar activity and the solar cycle (see also discussion in Heinzel et al. 2003a).

It can be seen in Fig. 8 that the maps of $h_{3}$ are more structured than the maps of $h_{4}$. This could be explained by the fact that the computed values of $h_{3}$ are more sensitive to intensity variations than those of $h_{4}$.

There were points inside the EUV-filament area where the model gave no results or the height values were greater than the prescribed limits $\left(50000 \mathrm{~km}\right.$ for $h_{3}$ and $74000 \mathrm{~km}$ for $h_{4}$ ). We have denoted those points as "black pixels". This can be seen in the original height maps in Fig. 9. To improve the presentation we used an interpolation between these points to construct new smoothed height maps which are shown in Fig. 8. The existence of points inside the EUV-filament area, where the model gave us no results, can be due to approximations like homogenous quiet-Sun intensities and scale-height values and/or due to noise in the observed data. The problem of the "black pixels" is discussed in detail in Sect. 6.1.

The interpolated (smoothed) height maps shown in Fig. 8, were finally used to construct a $3 \mathrm{D}$ visualisation of the EUV-extensions. For this we took maps computed for $\tau_{912}=5$. Resulting 3D structures are shown in Fig. 10 where various projections are taken. The projection in Fig. 10b resembles the pattern in Fig. 8. The yellow surface corresponds to the top surface, the violet one to the bottom surface of the EUV-extension. In order to make these surfaces well visible, we had to cut them artificially at some height above the solar surface, we took $h=8000 \mathrm{~km}$. The most interesting feature is a bending of both surfaces towards the solar surface. Such a shape could result from a magnetic arcade with twisted flux tubes as in the model of Anzer \& Heinzel (2003).

\subsection{Heights of upper and lower boundaries of the EUV-filament using $\tau_{912}$ computed from intensity measurements of the OV and OVI TR lines}

The method described in Sect. 3.2 was used to compute $\tau_{912}$ from $\mathrm{OV}$ and $\mathrm{OVI}$ transition-region lines. The $\mathrm{OV}$ line was observed by SoHO/CDS and the intensity map of this line is shown in the bottom image in Fig. 2. The SoHO/SUMER slit position $(X=122 \operatorname{arcsec}$ and $Y=59 \div 179$ arcsec in relative CDS-raster coordinates) is also displayed in this image by a vertical bar. Its dashed part crosses the EUV-filament area. The O VI line was observed by SUMER only at this slit position at a time close to the CDS observations. So we got the O VI line intensity values only for a cut through the EUV-filament and the surrounding quiet-Sun region.

The $\mathrm{OV}$ and $\mathrm{OVI}$ line intensities are shown in Fig. 3. Intensity values between $Y=69$ and $Y=90 \operatorname{arcsec}$ were used for the parameter $b$ estimate (see Eq. (15)). This part of the cut lying within the quiet-Sun region was most suitable for estimating $b$ because of the best correlation between the $\mathrm{OV}$ and $\mathrm{O}$ VI line intensities. The coefficient of correlation is equal to 0.94 for that range. We estimated a value of 9.7 for $b$ using the least-square method.

$I_{\mathrm{fg}}(\mathrm{OV})$ was estimated as an average value from the two largest and darkest $\mathrm{H} \alpha$ parts of the filament labeled I and II in the bottom image in Fig. 2. This average value is listed in the Table 1.

The range from $Y=98$ to $Y=157$ arcsec (hereafter called the "EUV-filament cut") was considered as part of the cut lying inside the EUV-filament area. For this part of the cut the values of $\tau_{912}$ were computed (using Eqs. (24) and (13)) for different values of the parameter $a$ and they are shown in Fig. 11. The parameter $a$ (see Eq. (20)) gives the ratio between the TR line intensity emitted from the PCTR1 (on top or bottom of the $\mathrm{H} \alpha$ parts of the filament) and from the PCTR2 (EUV-filament extensions). For example, there is no PCTR2 if $a$ is equal to zero. The method gave no results in some parts of the EUV-filament cut for $a$ greater than 0.57 (the expression in the logarithm of Eq. (24) was not positive). Therefore we have considered that value as an upper limit for the parameter $a$.

The heights of the upper and lower filament boundaries along the EUV-filament cut were then computed using the $\tau_{912}$ values plotted in Fig. 11 for different values of the parameter $a$. These heights are shown in Fig. 12. The spectroscopic model described in Sect. 3.1 was used for this computation. For the quantities $I_{\mathrm{qs}}$ and $I_{\mathrm{fg}}$ of the $\mathrm{MgX}$ and Si XII lines and for the angle $\alpha$ between the line of sight and radial direction, the same values as in Sect. 4.1, were used (Table 1, and $\alpha=38^{\circ} .9$ ). The same height limits $\left(h_{3}<50000 \mathrm{~km}\right.$ and $\left.h_{4}<74000 \mathrm{~km}\right)$ were imposed as in Sect. 4.1. Again, we found points ("black pixels") where the model gave no results and these points were interpolated in Fig. 12. This seems to indicate that the "black pixels" occurrence in the original height maps (Fig. 9) is not due to a unique $\tau_{912}$ value for the whole EUV-filament area.

To verify the reliability of our model it is important to estimate how any deviations in intensity measurements could influence the calculated heights $h_{3}, h_{4}$. A simple test of stability 
$h_{3}[\mathrm{~km}] ; \quad \tau(912 \AA)=5.0$

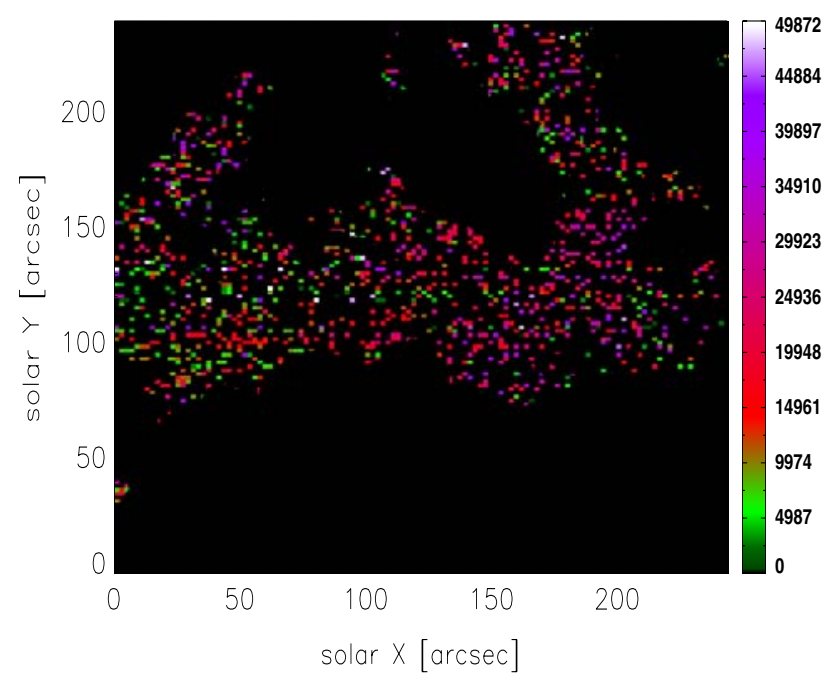

$h_{4}[\mathrm{~km}] ; \quad \tau(912 \AA)=5.0$

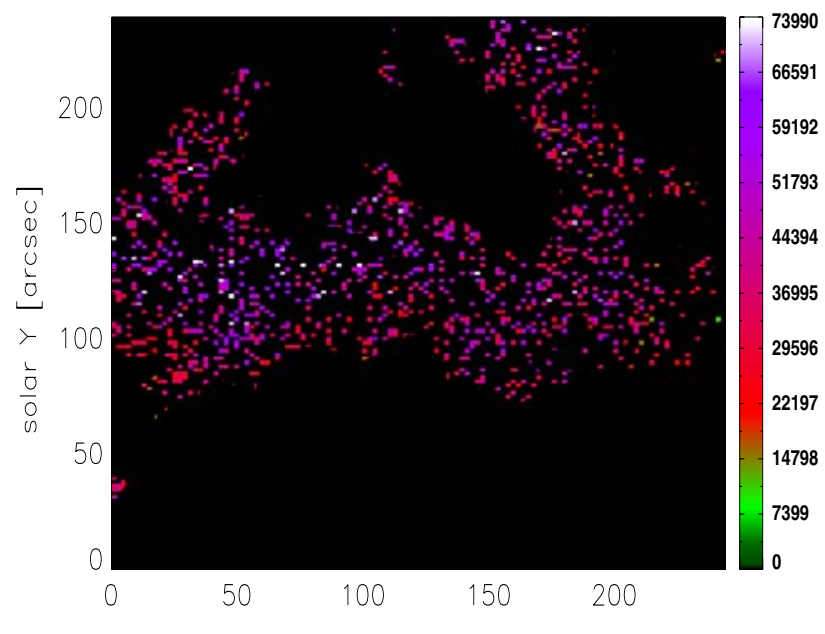

solar $X[\operatorname{arcsec}]$

Fig. 9. Original height maps (before interpolation) of the EUV-filament extensions using the model of Heinzel et al. (2003a) for $\tau_{912}=5.0$ Note many "black pixels" inside the EUV-filament.

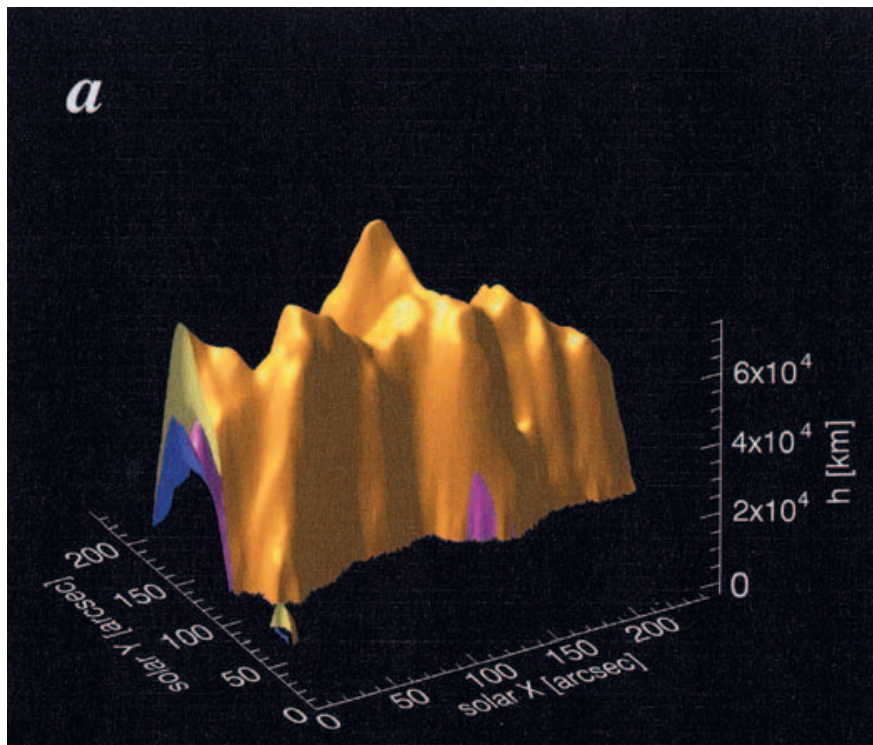

c

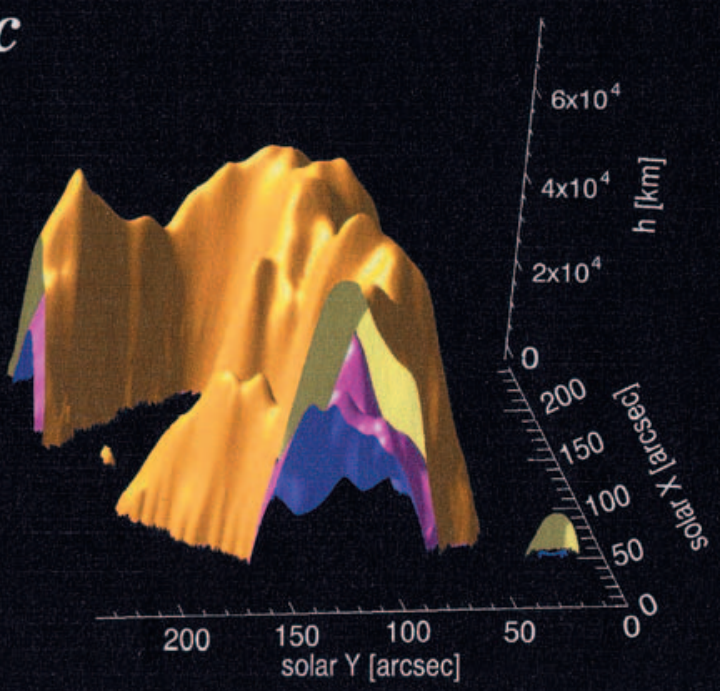

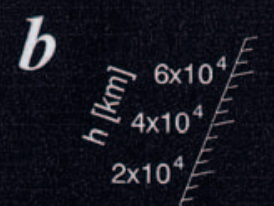

b

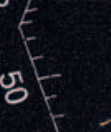

웅

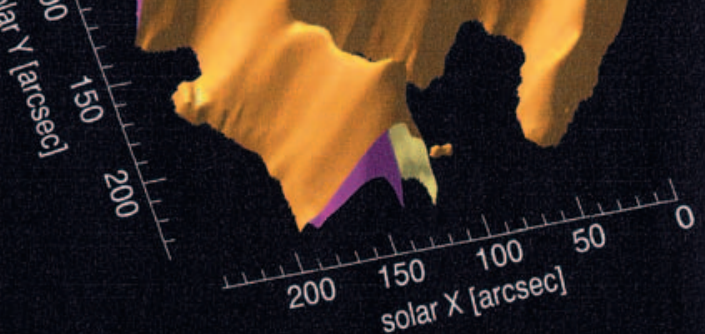

d

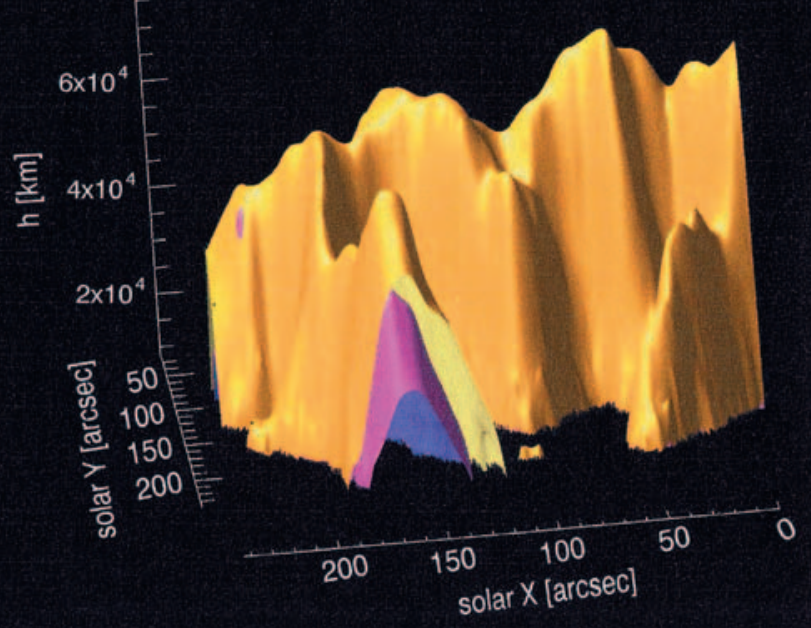

Fig. 10. 3D visualisations of the EUV-extension structure computed for $\tau_{912}=5.0$ shown from four different view-angles. 


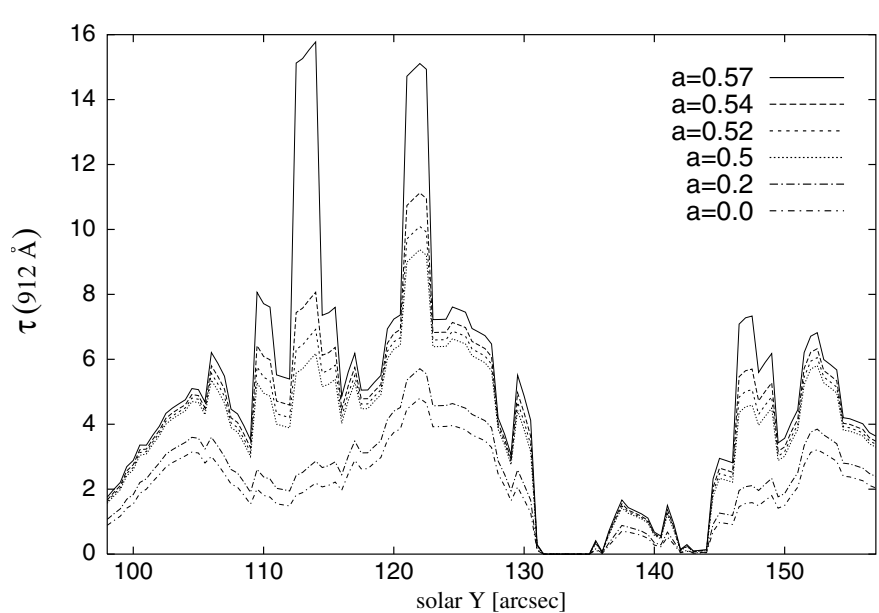

Fig. 11. Values of $\tau_{912}$ for the EUV-filament cut computed from O V and $\mathrm{O}$ VI line intensities for different values of $a$.

was already made by Heinzel et al. (2003a) and the results are shown in their Table II. From the values in that table two conclusions can be made for result reliability:

1. A small intensity variation of one or both lines by $\pm 5 \%$ can cause rather large deviations of $h_{3}$ up to $73 \%$ but $h_{4}$ is changing only by $2 \%$ up to $18 \%$.

2. One can see that the calculated heights are slightly more sensitive to intensity variations of the $\mathrm{MgX}$ line than to those of the Si XII line.

We have made now a more complex analysis of the stability of the model calculations. We examined stability calculations for five values of $\tau_{912}(1.0,3.0,5.0,7.0$ and 8.0) and for three height pairs $h_{3}, h_{4}$ (representing lower, medium and larger altitudes):

$h_{3} \approx 10000 \mathrm{~km} \quad h_{4} \approx 20000 \mathrm{~km}$,

$h_{3} \approx 20000 \mathrm{~km} \quad h_{4} \approx 40000 \mathrm{~km}$,

$h_{3} \approx 40000 \mathrm{~km} \quad h_{4} \approx 60000 \mathrm{~km}$.

Similarly to Heinzel et al. (2003a) we also found that $h_{3}$ is much more sensitive to intensity variations than $h_{4}$ and the calculations are slightly more sensitive to variations of the Si XII line intensity compared to the $\mathrm{MgX}$ line. Positive variations of the $\mathrm{MgX}$ intensity cause negative variations of heights but for variations of the Si XII line intensity the situation is opposite. The model sensitivity to intensity variations for $\tau_{912}=5$ and medium heights is shown in Fig. 13 .

For $\tau_{912}=5$ and medium height values, the $\mathrm{MgX}$ line intensity variation of $+5 \%$ leads to computed $h_{3}$ and $h_{4}$ values which are by $45 \%$ and $10 \%$ lower, respectively. The sensitivity of $h_{3}$ is almost constant for $\tau_{912}$ changes but the sensitivity of $h_{4}$ slowly decreases with the rise of $\tau_{912}$. For larger heights and low intensity values, the heights are less sensitive to intensity variations. For example, for values $h_{3} \approx 40000 \mathrm{~km}, h_{4} \approx$ $60000 \mathrm{~km}$ and $\tau_{912}=5$, a variation of the $\mathrm{Mg} \mathrm{X}$ line intensity by $+5 \%$ causes a $30 \%$ lower $h_{3}$ and a $8 \%$ lower $h_{4}$. For lower height values and large intensity values, $h_{3}$ is more sensitive to intensity variations than for medium heights. $h_{4}$ is only somewhat more sensitive. For example for heights $h_{3} \approx 10000 \mathrm{~km}$, $h_{4} \approx 20000 \mathrm{~km}$ and $\tau_{912}=5$, calculated heights $h_{3}$ and $h_{4}$ are by $65 \%$ and $12 \%$ lower, respectively, if the $\mathrm{Mg} \mathrm{X}$ line intensity is changed by $+5 \%$.

\section{Stability of spectroscopic model calculations}

A brief overview of height value errors is shown in Table 2. In the first and third columns the relative intensities of the $\mathrm{MgX}$ and Si XII lines are listed. In the second and fourth columns the errors of intensity measurements are listed. Values of these errors were estimated assuming calibration linearity and Poisson statistics for detected counts (Thompson 2000). In the last four columns the heights and their relative errors are listed. Combinations of the upper and lower heights listed in this table were chosen, from computed $\tau_{912}=5$ pairs of $h_{3}$ and $h_{4}$ values (shown in Fig. 9), according to the following key: We chose $h_{3}$ values from six groups: below $8000 \mathrm{~km}$ and around the heights 10000, 20000, 30000, 40000 and $50000 \mathrm{~km}$. Both the lowest and highest $h_{4}$ values were complemented to the $h_{3}$ values chosen from these six groups. So, one can imagine how the height errors are changing with heights and intensities. The height errors on the left side from the slash symbol correspond to intensities lowered by errors of intensity measurements (listed in the second and fourth columns). The height errors on the right side from the slash symbol correspond to intensity values increased by errors of intensity measurements. For the $\mathrm{Mg} \mathrm{X}$ and Si XII line intensities deviating in opposite directions the model gives no solution. This could be one of the "black pixels" sources. The general trend of decreasing height errors with increasing heights can be seen in Table 2.

\section{General behaviour of the spectroscopic model}

\subsection{Reasons for the existence of "black pixels"}

As was already said in the last paragraph of the previous section the noise in intensity measurements seems to be one of the reasons for the "black pixels" occurrence. But the noise in $\mathrm{Mg} \mathrm{X}$ and Si XII intensity data cannot be the only reason for the occurrence of the "black pixels" because there are many such points where the intensities are large enough so that the noise can be neglected and there is still no solution. We found that the majority of these points appears the same on height maps computed for $\tau_{912}$ values of 3.0-8.0. Even more, the black pixels occurred in the case when $\tau_{912}$ was computed from $\mathrm{O} v$ and O VI TR line intensities (but the black pixels were interpolated in the height plots shown in Fig. 12). By overlying maps computed for different $\tau_{912}$ values we could see that the general pattern remains similar. This led us to the conclusion that the presence of "black pixels" is not due to the assumption of a uniform optical thickness in the whole area.

The occurrence of the "black pixels" could also be explained by the fact that we used unique values of the quietSun intensity and unique values of scale height for both coronal lines. So we took the same quiet-corona intensity dependence on height (Eq. (3); $I_{\mathrm{o}}$ is connected with $I_{\mathrm{qs}}$ through Eqs. (2) and (4)) for the whole EUV-filament area. Some uncertainties still remain, i.e. the large altitudes in Fig. 12 between pixels 130 and 140. 

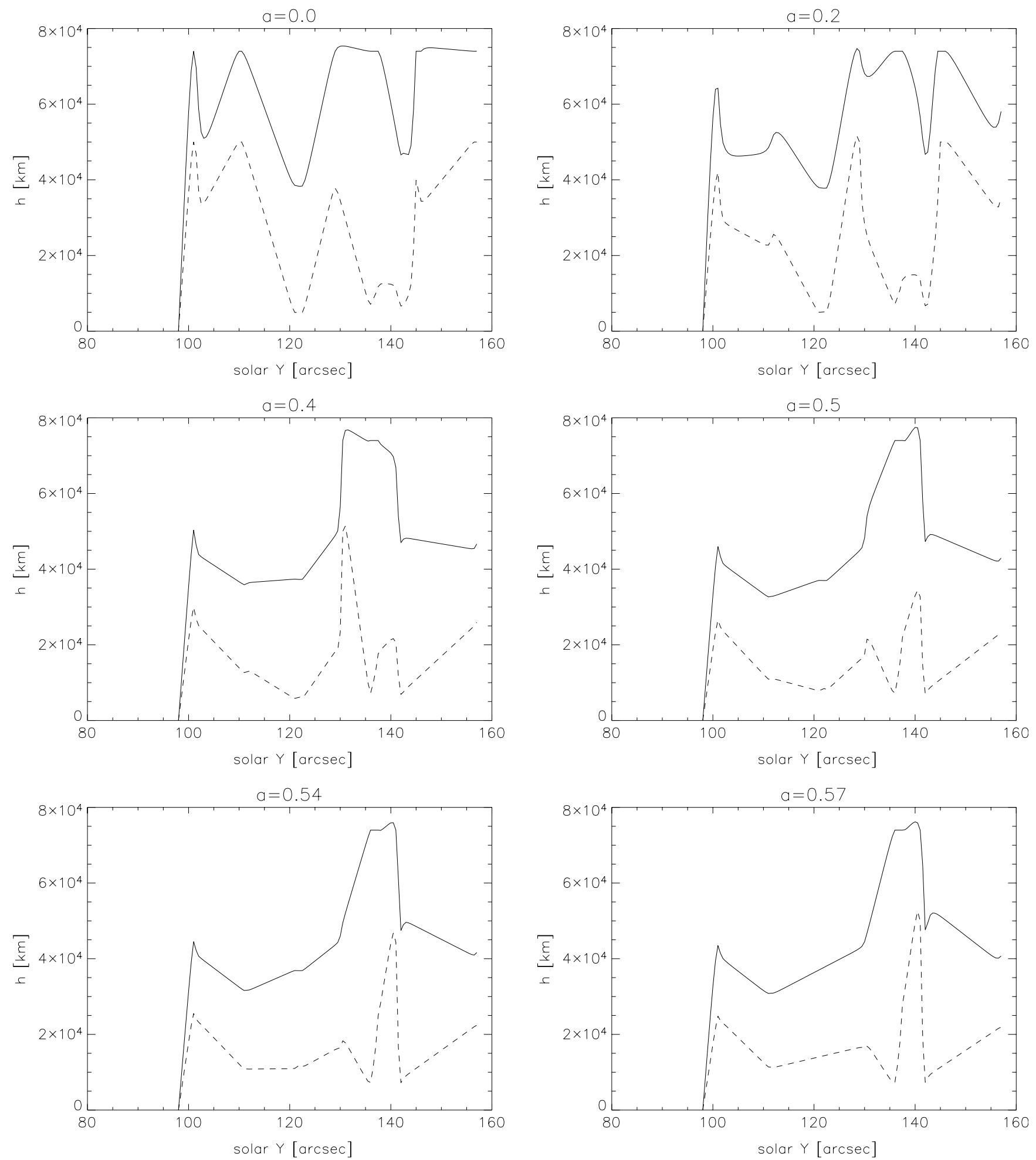

Fig. 12. Plots of heights along the EUV-filament cut using $\tau_{912}$ computed for different values of the parameter $a$ (see Fig. 11). Height distributions along the EUV-filament cut are almost independent of the variation of $a$ between 0.54 and 0.57 . Values of $h_{4}$ are plotted by full line and values of $h_{3}$ by dashed line.

In Fig. 14, gray areas show that a solution exists only for some combinations of the $\mathrm{Mg} \mathrm{X}$ and Si XII line intensities. These areas were computed for three combinations of scaleheight values using the forward method (Eq. (9)). It is shown in this figure that if the scale-height of the $\mathrm{Mg} \mathrm{X}$ line is changing in the range from 20000 to $50000 \mathrm{~km}$ and the scale-height of Si XII in the range from 30000 to $50000 \mathrm{~km}$, there exist solutions in a majority of points which are gathered in an area of greatest density of observed points. Other observed points are more scattered in this plot. 

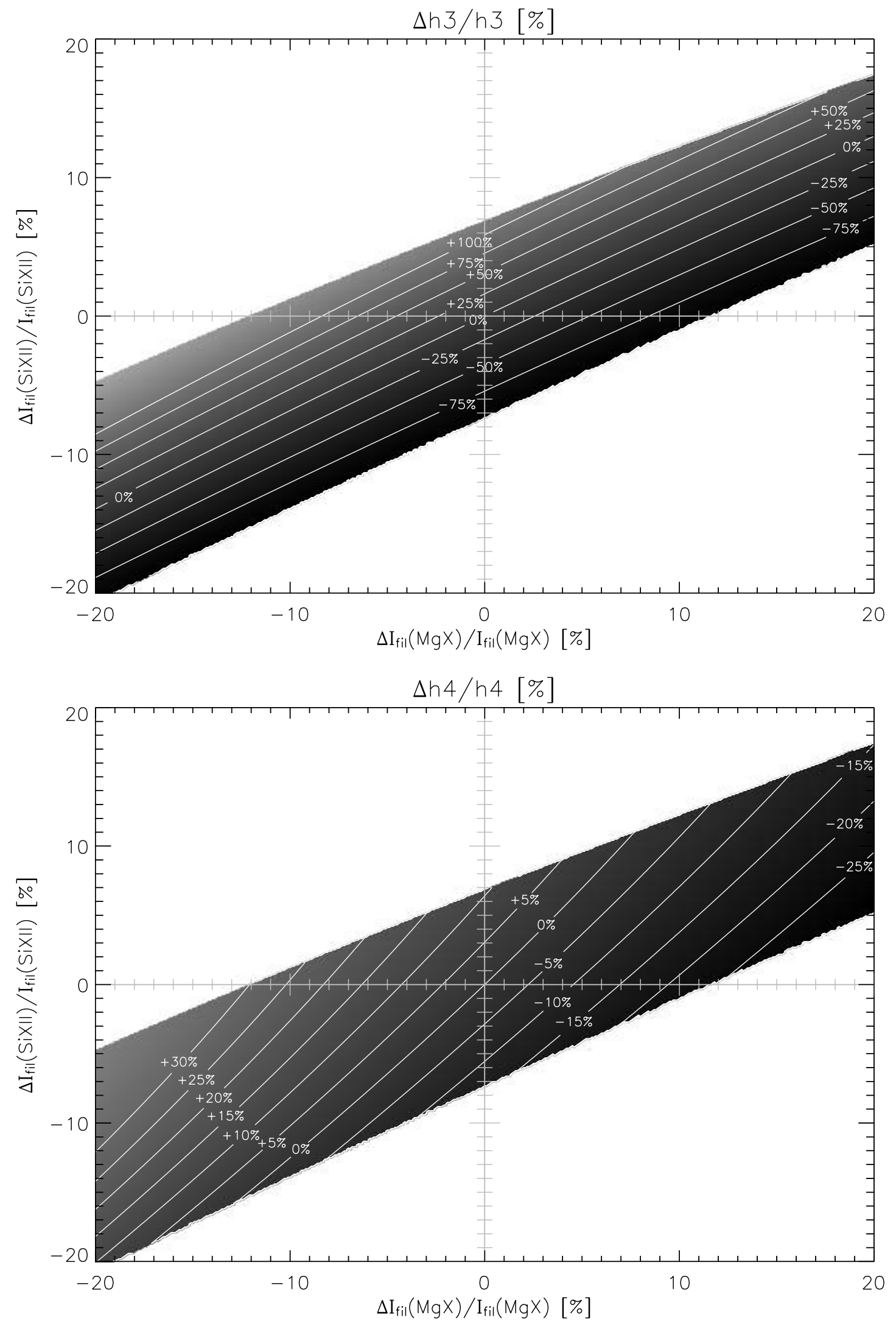

Fig. 13. Plots showing the stability of the spectroscopic model taking $\tau_{912}=5$ for both coronal line intensity variations. The heights are $h_{3} \approx$ $20000 \mathrm{~km}, h_{4} \approx 40000 \mathrm{~km}$ with the intensities $I_{\text {fil }}(\mathrm{Mg} \mathrm{X})=31 \mathrm{erg} \mathrm{cm}^{-2} \mathrm{~s}^{-1} \mathrm{sr}^{-1}$ and $I_{\text {fil }}(\mathrm{Si} \mathrm{XII})=51 \mathrm{erg} \mathrm{cm}^{-2} \mathrm{~s}^{-1} \mathrm{sr}^{-1}$. Contours mark relative height deviation $(\Delta h / h)$ for both $h_{3}$ and $h_{4}$. Plots were drawn using the spectroscopic model of Heinzel et al. (2003a). 
Table 2. Brief overview of statistical errors of height values. More information about this table can be found in the last paragraph of Sect. 5 .

\begin{tabular}{|c|c|c|c|c|c|c|c|}
\hline$\frac{I_{\mathrm{fl} 1}(\mathrm{MgX})}{I_{\mathrm{qs}}(\mathrm{Mg} \mathrm{X})}$ & $\begin{array}{c}\frac{\Delta I_{\text {fil }}(\mathrm{Mg} \mathrm{x})}{I_{\text {fil }}(\mathrm{Mg} \mathrm{x})} \\
{[\%]}\end{array}$ & $\frac{I_{\mathrm{fil}}(\mathrm{SiXII})}{I_{\mathrm{qS}}(\mathrm{Si} \mathrm{XII)}}$ & $\begin{array}{c}\frac{\Delta I_{\text {fil }}(\mathrm{Si} \mathrm{XII})}{I_{\mathrm{fil}}(\mathrm{Si} \mathrm{XII})} \\
{[\%]}\end{array}$ & $\begin{array}{r}h_{3} \\
{[\mathrm{~km}]}\end{array}$ & $\begin{array}{c}\Delta h_{3} / h_{3} \\
{[\%]}\end{array}$ & {$[\mathrm{km}]$} & $\begin{array}{c}\Delta h_{4} / h_{4} \\
{[\%]}\end{array}$ \\
\hline 0.77 & $\mp 18$ & 0.84 & $\mp 13$ & 2900 & $+30 /+46$ & 11100 & $+76 /-60$ \\
\hline 0.20 & $\mp 21$ & 0.33 & $\mp 17$ & 5600 & $-83 /+100$ & 68600 & $+9 /-8$ \\
\hline 0.69 & $\mp 17$ & 0.81 & $\mp 13$ & 10900 & $-31 /+49$ & 16500 & $+44 /-36$ \\
\hline 0.21 & $\mp 20$ & 0.36 & $\mp 16$ & 13300 & $-47 /+54$ & 70900 & $+9 /-8$ \\
\hline 0.59 & $\mp 17$ & 0.75 & $\mp 15$ & 19700 & $-54 /+69$ & 24400 & $+24 /-21$ \\
\hline 0.22 & $\mp 24$ & 0.38 & $\mp 17$ & 19300 & $+6 /+16$ & 70600 & $+22 /-14$ \\
\hline 0.48 & $\mp 19$ & 0.67 & $\mp 14$ & 29900 & $-20 /+30$ & 35700 & $+24 /-19$ \\
\hline 0.25 & $\mp 22$ & 0.43 & $\mp 16$ & 28800 & $+2 /+12$ & 68400 & $+21 /-14$ \\
\hline 0.41 & $\mp 16$ & 0.62 & $\mp 13$ & 40600 & $-25 /+33$ & 45200 & $+15 /-13$ \\
\hline 0.25 & $\mp 18$ & 0.44 & $\mp 13$ & 38600 & $-5 /+14$ & 71300 & $+16 /-12$ \\
\hline 0.34 & $\mp 22$ & 0.56 & $\mp 15$ & 48700 & $+7 /+6$ & 55500 & $+28 /-19$ \\
\hline 0.26 & $\mp 20$ & 0.47 & $\mp 19$ & 48800 & $-42 /+56$ & 71900 & $+20 /-10$ \\
\hline
\end{tabular}
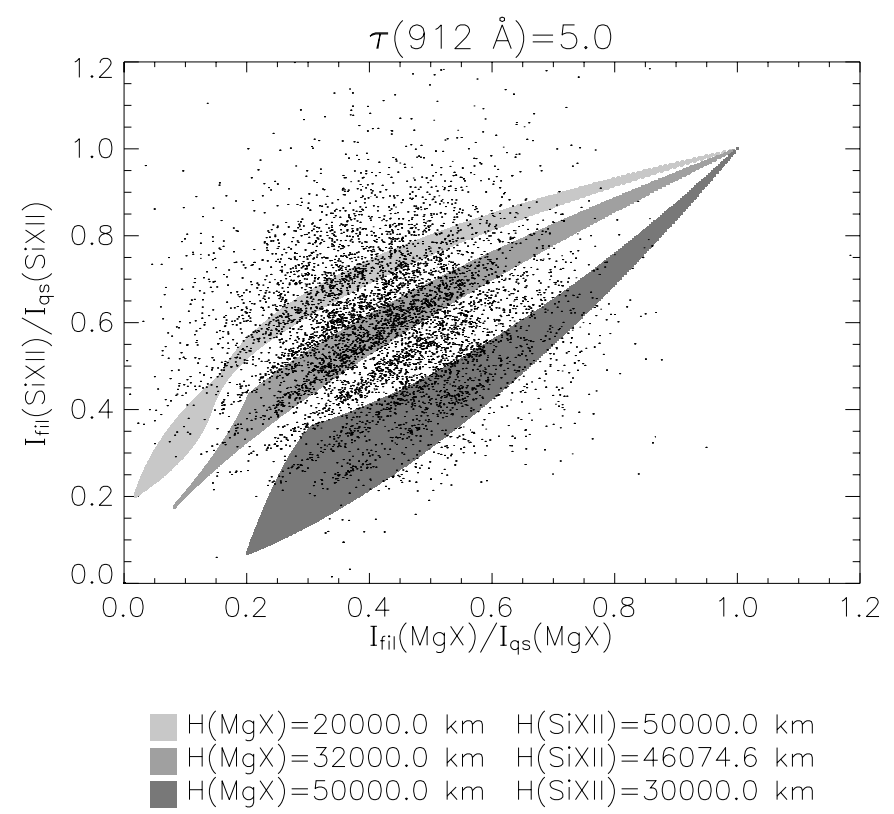

Fig. 14. Areas marking for which $\mathrm{MgX}$ and Si XII line intensities the model has a solution. These areas were computed for three combinations of scale-height values. Tiny black points give line intensities in all individual pixels of the CDS raster of the EUV-filament area.

For our computations of the height maps (Fig. 8) we used the scale heights $H(\mathrm{MgX})=32000 \mathrm{~km}$ and $H(\mathrm{Si} \mathrm{XII})=$ $46074.6 \mathrm{~km}$. For this combination the middle area in Fig. 14 indicates the range of solutions. All observed points outside this middle area correspond in fact to "black pixels" (about $70 \%$ of all observed points). Note finally that if $I_{\mathrm{qs}}$ is uniform, the solutions in Fig. 14 are independent of its actual value (we use normalized intensities).

\subsection{Relative importance of absorption and volume blocking}

Comparing the influence of volume blocking and hydrogen Lyman-continuum absorption by the EUV-filament plasma on coronal line intensities one can estimate the EUV-filament material characteristics. The decrease of intensity of coronal radiation due to the presence of the EUV-filament (because of absorption and volume blocking) can be derived from Eq. (9):

$\frac{I_{\mathrm{qs}}-I_{\mathrm{fil}}}{I_{\mathrm{qs}}}=f\left(h_{4}\right)-f\left(h_{3}\right) \exp \left(-\tau_{\lambda}\right)$.

The intensity decrease due to volume blocking only can be computed from Eq. (26) by simply setting $\tau_{\lambda}=0$ :

$\left[\frac{I_{\mathrm{qs}}-I_{\mathrm{fil}}}{I_{\mathrm{qs}}}\right]_{\mathrm{vb}}=f\left(h_{4}\right)-f\left(h_{3}\right)$.

The intensity decrease due to absorption only can be found by subtracting Eq. (27) from Eq. (26):

$\left[\frac{I_{\mathrm{qs}}-I_{\mathrm{fil}}}{I_{\mathrm{qs}}}\right]_{\mathrm{abs}}=f\left(h_{3}\right)\left[1-\exp \left(-\tau_{\lambda}\right)\right]$.

The ratio between intensity decrease due to absorption and due to volume blocking is shown in the plots in Fig. 15 for both $\mathrm{MgX}$ and Si XII lines and for $\tau_{912}=5$. For heights $h_{3}$ and $h_{4}$ in the area under the contour labeled 1.0, the hydrogen Lyman-continuum absorption by the EUV-filament plasma is low and the intensity decrease is mainly due to volume blocking. But in the area above that contour the absorption is remarkable. Large ratios are represented by contours on which $h_{3}$ reaches $h_{4}$. This means that geometrical thickness of the filament reaches zero $(\Delta h \rightarrow 0)$ and there is no space for volume blocking. Plots for different $\tau_{912}$ values look very similar to those in Fig. 15 drawn for $\tau_{912}=5$. But the contours increase with $h_{4}$ slightly less steeply for $\tau_{912}$ larger than 5 .

It is important for the characteristics of the EUV-filament material to compare the total area where absorption dominates 

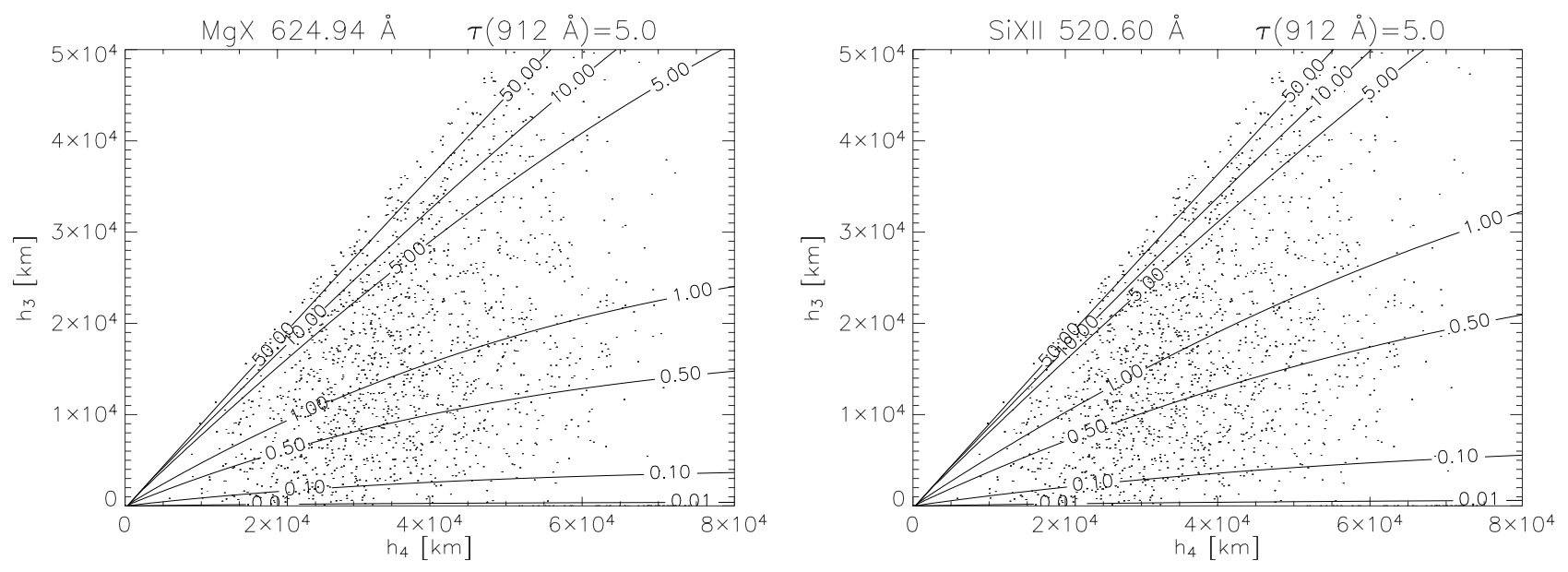

Fig. 15. Contours of the ratio between intensity decrease due to absorption to that due to volume blocking, in the $h_{4}$ versus $h_{3}$ plot for the Mg X and Si XII lines and for $\tau_{912}=5.0$. Tiny black points are heights resulting from spectroscopic models for pixels of the CDS raster from the EUV-filament area without any interpolation of "black pixels".

to the total area where volume blocking is the principal reason of intensity decrease. This is made by comparing the number of CDS raster pixels with absorption predominance and the umber of pixels with the volume-blocking predominance. Therefore we counted the points over and under the contour labeled 1.0 in the plots in Fig. 15 and these numbers were divided by the total number of points. Then these relative numbers of points, where absorption or volume blocking dominates, were denoted as $n_{\mathrm{pts}}(\mathrm{abs})$ and $n_{\mathrm{pts}}(\mathrm{vb})$, respectively.

There is approximately the same number of pixels with the absorption and the volume blocking predominance for $\tau_{912} \approx 2$ for the $\mathrm{Mg}$ X line. When $\tau_{912}$ goes to $0, n_{\mathrm{pts}}(\mathrm{abs})$ and $n_{\mathrm{pts}}(\mathrm{vb})$ go steeply to 0.0 and to 1.0 , respectively. There is a less steep $n_{\mathrm{pts}}(\mathrm{abs})$ rise and $n_{\mathrm{pts}}(\mathrm{vb})$ decrease when $\tau_{912}$ is rising. For example, relative numbers of points for $\tau_{912}=12$ are: $n_{\mathrm{pts}}(\mathrm{abs})=$ 0.67 and $n_{\mathrm{pts}}(\mathrm{vb})=0.33$. For the Si XII line there would be the same values of $n_{\text {pts }}(\mathrm{abs})$ and $n_{\mathrm{pts}}(\mathrm{vb})$ at $\tau_{912}=11.3$. The value of $n_{\mathrm{pts}}(\mathrm{abs})$ decreases to 0.0 and the value of $n_{\mathrm{pts}}(\mathrm{vb})$ rises to 1.0 with rising $\tau_{912}$ but not as steeply as for the $\mathrm{Mg} \mathrm{X}$ line. The values of $n_{\mathrm{pts}}(\mathrm{abs})$ and $n_{\mathrm{pts}}(\mathrm{vb})$ are almost the same for $\tau_{912}=12$ as for the $\mathrm{Mg} \mathrm{X}$ line.

\section{Discussion and conclusions}

The most important result of this study is the determination of the 3D structure of the EUV-extensions of the filament observed on 15 October 1999. For this we used the SoHO/CDS and SoHO/SUMER observations, together with $\mathrm{H} \alpha$ images from VTT/MSDP. To derive the filament altitudes, we used the spectroscopic model of Heinzel et al. (2003a), taking into account the projection effects (using the plane-parallel approximation) because the filament was not located at the disk center. We found that the EUV-filament extensions are located at high altitudes, sometimes larger than $70000 \mathrm{~km}$. This result is rather robust, irrespectively of various uncertainties discussed in the text (like data noise, values of $\tau_{912}$ or the problem of "black pixels"). From the distribution of derived heights $h_{3}$ and $h_{4}$ in Fig. 15 we estimated mean values $h_{3}=15000 \mathrm{~km}$ and $h_{4}=40000 \mathrm{~km}$. However, there are significant variations around these representative values and we see from Fig. 15 that in about half of observed pixels the Lyman-continuum absorption dominates in the coronal-line depression, while in the other half it is the volume blocking effect. The latter situation corresponds to large (larger than $30000 \mathrm{~km}$ ) geometrical thickness of the EUV-filament extension, i.e. to very large distance between yellow and violet surfaces in Fig. 10.

What can still be questionable is a proper determination of the EUV-filament extensions and their boundaries. In this paper we have defined the location of the EUV-filament extensions by taking a certain level of image contrast relative to a mean "quiet-Sun" area. This, however, may include some regions which in fact do not belong to the extended EUV-filament but rather to the surrounding disk areas. Then it may happen that great altitudes are derived from our spectroscopic model for such dark areas if the scale heights and the actual value of the quiet-Sun intensity favour such solutions. In other words, the spectroscopic model does not prove the existence of an EUV-filament at a given position, but rather gives a reasonable estimate of altitudes at places which are supposed to belong to the EUV-filament.

A more rigorous way of determining the EUV-filament contours would be to use two rasters simultaneously obtained in two similar TR lines, where one was observed at the wavelength below $912 \AA$ and the other one at the wavelength above $912 \AA$. An example of such a line pair is O V and O VI discussed in this paper. This would clearly discriminate between the dark features belonging to the EUV-filaments (absorption in $\mathrm{OV}$ ) and those pertinent to the transition region outside the filament (similar behaviour of both lines). In Fig. 3 we show a cut through the filament along which the intensities of these two lines are plotted. While the $\mathrm{O}$ VI line exhibits similar intensity variations along the whole cut, the $\mathrm{OV}$ line is significantly depressed in the region of the EUV-filament. This confirms the behaviour described by Chiuderi et al. (2001) who found that the EUV-filaments are invisible in TR lines longward of $912 \AA$. Using these results, we see that at least along the SUMER slit 
cut the EUV-filament boundary is consistent with that derived independently from our contrast considerations.

Our finding that EUV-extensions are located at great altitudes supports the concept of Anzer \& Heinzel (2003) who suggested that such structures can be modeled as vertically extended systems of twisted flux tubes in which the filament plasma is located in shallow magnetic dips. On the other hand, the low-lying parasitic dips suggested by Aulanier \& Schmieder (2002) should be visible due to a volume blocking effect even in lines like the O VI, provided that the scale height of such TR lines is comparable to the altitudes of parasitic dips $(<4000 \mathrm{~km})$. So far, no intensity depressions were seen in TR lines above $912 \AA$ (Chiuderi et al. 2001; our Fig. 3) and thus this issue deserves further attention. In particular, it is important to obtain a precise measurements of TR-line intensity variations close to the limb and derive realistic scale heights (see e.g. the behaviour of the Ov intensity in Fludra et al. 1999).

The results of this study were used by Heinzel et al. (2003b) to estimate the mass loading of the extended parts of the EUV-filament. Using the approximate formulas derived in Heinzel et al. (2003b), the plasma density was computed at each pixel position of the CDS raster and then multiplied by the pixel size and filament thickness which comes from Fig. 8. This was done for values of $\tau_{912}$ from 2 to 5 . Then the average density of the EUV-extensions ranged from $3.9 \times 10^{-15}$ to $6.3 \times 10^{-15} \mathrm{~g} \mathrm{~cm}^{-3}$ and this has to be compared with densities from $2 \times 10^{-14}$ to $2 \times 10^{-12} \mathrm{~g} \mathrm{~cm}^{-3}$ typical for quiescent prominences (Jensen \& Wiik 1990). The resulting mass is in the range from $8.1 \times 10^{14}$ to $1.5 \times 10^{15} \mathrm{~g}$ which is comparable to the mass of coronal mass ejections (Simnett 2000; Webb 2000).

In a forthcoming paper we plan to perform detailed nonLTE computations of the hydrogen ionization structure inside the EUV-filament extensions and then evaluate the mass loading in detail. Such calculations will also provide us with the mass of those parts of the filament visible in $\mathrm{H} \alpha$ and this will finally allow us to make conclusions about the mass loading in the whole filament.

Acknowledgements. SoHO is a space mission of international cooperation between ESA and NASA. This work was partly supported by grants A3003203 and S-1003006 of the Grant Agency of the Academy of Sciences of the Czech Republic and the key project K2043105. SoHO CDS and SUMER observations were obtained during coordinated MEDOC, THEMIS, and VTT campaigns (JOPs 17/107, 95 and 131). We thank all observers and SoHO planners. We also thank Dr. N. Mein and G. Mező for reduction of VTT/MSDP data and Dr. J. Rybak for providing us with the code for drawing the color scaled maps. P.S. acknowledges the support of Astronomical Institute of Academy of Sciences of the Czech Republic during his postdoctoral stay. P.H. and B.S. are members of the European Solar Magnetism Network (ESMN-HPRN-CT-2002-00313) from which this work was partially supported. Finally, we are indebted to the referee for constructive comments and suggestions.

\section{References}

Anzer, U., \& Heinzel, P. 2003, A\&A, 404, 1139

Arnaud, M., \& Raimond, J. C. 1992, ApJ, 398, 39

Aulanier, G., \& Schmieder, B. 2002, A\&A, 386, 1106

Chiuderi Drago, F., Alissandrakis, C. E., Bastian, T., Bocchialini, K., \& Harrison, R. A. 2001, Sol. Phys., 199, 115

Fludra, A., del Zanna, G., Alexander, D., \& Bromage, B. J. I. 1999, J. Geophys. Res., 104, No. A5, 9709

Fludra, A. 2003, private communication

Griem, H. R. 1997, Principles of Plasma Spectroscopy (Cambridge: Cambridge University Press), 241

Harrison, R. A., Sawyer, E. C., Carter, M. K., et al. 1995, Sol. Phys., 162,233

Heinzel, P., Schmieder, B., \& Tziotziou, K. 2001, ApJ, 561, L223

Heinzel, P., Anzer, U., \& Schmieder, B. 2003a, Sol. Phys., 216, 159

Heinzel, P., Anzer, U., Schmieder, B., \& Schwartz, P. 2003b, EUV-filaments and their mass loading, in International solar cycle studies Symp. 2003, ed. A. Wilson, ESA SP-535, 447

Jensen, E., \& Wiik, J. E. 1990, Plasma parameters in quiescent prominences, in Dynamics of quiescent prominences, ed. V. Ruždjak, \& E. Tandberg-Hanssen (New York: Springer-Verlag), Proc. IAU Coll., 117, 298

Kohl, J. L., Noci, G., Antonucci, E., et al. 1997, Sol. Phys., 175, 613

Schmieder, B., Tziotziou, K., \& Heinzel, P. 2003, A\&A, 401, 361

Schwartz, P. 2002, Iron Abundance in the solar corona determined from the photometry of green coronal line, $\mathrm{Ph} . \mathrm{D}$. Thesis (in Slovak), Astronomical Institute of Slovak Academy of Sciences, Tatranská Lomnica, Slovakia, 27

Simnett, G. M. 2000, J. Atm. Sol.-Terr. Phys., 62, No. 16, 1479

Sterling, A. C., Pike, C. D., Mason, H. E., Watanabe, T., \& Antionchos, S. K. 1999, ApJ, 524, 1096

Thompson, W. 2000, Deriving statistics from NIS data, CDS SOFTWARE NOTE, 49, version 5

Young, P. R., del Zanna, G., Landi, E., et al. 2003, ApJS, 144, 135

Webb, D. F. 2000, J. Atm. Sol.-Terr. Phys., 62, No. 16, 1415

Wilhelm, K., Curdt, W., Marsch, E., et al. 1995, Sol. Phys., 162, 189 